\title{
The design and performance of a prototype water Cherenkov optical time-projection chamber
}

\author{
Eric Oberla, Henry J. Frisch
}

Enrico Fermi Institute, University of Chicago; 5640 S. Ellis Ave., Chicago IL, 60637

\begin{abstract}
A first experimental test of tracking relativistic charged particles by 'drifting' Cherenkov photons in a water-based optical time-projection chamber (OTPC) has been performed at the Fermilab Test Beam Facility. The prototype OTPC detector consists of a $77 \mathrm{~cm}$ long, $28 \mathrm{~cm}$ diameter, $40 \mathrm{~kg}$ cylindrical water mass instrumented with a combination of commercial $5.1 \times 5.1 \mathrm{~cm}^{2}$ micro-channel plate photo-multipliers (MCPPMT) and $6.7 \times 6.7 \mathrm{~cm}^{2}$ mirrors. Five MCP-PMTs are installed in two columns along the OTPC cylinder in a small-angle stereo configuration. A mirror is mounted opposite each MCP-PMT on the inner surface of the detector cylinder, effectively increasing the photo-detection efficiency and providing a time-resolved image of the Cherenkov light on the opposing wall. Each MCP-PMT is coupled to an anode readout consisting of thirty $50 \Omega$ microstrips. A 180-channel data acquisition system digitizes the MCP-PMT signals on one end of the microstrips using the PSEC4 waveform sampling-and-digitizing chip operating at a sampling rate of 10.24 Gigasamples-per-second. The single-ended microstrip readout determines the time and position of a photon arrival at the face of the MCP-PMT by recording both the direct signal and the pulse reflected from the unterminated far end of the strip . The detector was installed on the Fermilab MCenter secondary beam-line behind a steel absorber where the primary flux is multi-GeV muons. Approximately 80 Cherenkov photons are detected for a through-going muon track in a total event duration of $\sim 2 \mathrm{~ns}$. By measuring the time-of-arrival and the position of individual photons at the surface of the detector to $\leq 100 \mathrm{ps}$ and a few $\mathrm{mm}$, respectively, we have measured a spatial resolution of $\sim 15 \mathrm{~mm}$ for each MCP-PMT track segment, and, from linear fits over the entire track length of $\sim 40 \mathrm{~cm}$, an angular resolution on the track direction of $\sim 60 \mathrm{mrad}$.
\end{abstract}

Keywords:

Time-of-Flight; Cherenkov light; Optical Time Projection Chamber; Microchannel Plate Photomultiplier; track reconstruction; particle detector

\section{Introduction}

We report the design, construction, and performance of a small prototype 'Optical Time Projection 3 Chamber (OTPC) consisting of $40 \mathrm{~kg}$ of water viewed by five micro-channel plate photomultipliers (MCP${ }_{4}$ PMTs). The principle of the OTPC is that with photodetectors with adequate time and space resolution, 5 measuring the time and position of arrival of each individual photon emitted by Cherenkov radiation [1]

Email address: ejo@uchicago.edu (Eric Oberla) 
from charged particles traversing a volume allows the measurement of the photon drift times. Given the arrival positions and the drift times, the track position and direction can be reconstructed, in analogy with reconstructing tracks from the electron drift times in a conventional gaseous or noble liquid TPC.

Due to the faster drift velocity in a medium of photons compared to that of electrons, the expected spatial resolution measured by timing will be poorer. However, the scale for the required spatial resolution for a large water neutrino detector, for example, is set by the radiation length in water, which is approximately $40 \mathrm{~cm}$ [2]. A resolution of less than a few $\mathrm{cm}$ per optical sensor may then be adequate to do event tracking for isolated or separated tracks.

The OTPC rests on three enabling technologies:

1. the use of MCP-based photodetectors with a single photo-electron transit-time resolution measured in tens of picoseconds and a correlated position measurement with a resolution measured in mm [3, 4, 5, 6], able to cover a large surface area economically. In a conventional large water Cherenkov detector the size of the PMT precludes knowing the position of arrival to within the size of the cathode.

2. The use of microstrip anodes [7, 8, with bandwidths matched to the photodetector pulse, to cover large areas while preserving the temporal and spatial information encoded by the incident photon. The microstrips provide spatial resolution along the strip direction from the difference in pulse arrival times at the two ends, and in the transverse direction by charge sharing among strips, allowing mm resolution in two dimensions while requiring only a one-dimensional array of electronics readout channels [9].

3. The development of an economical (in quantity) low-power waveform sampling Application Specific Integrated Circuit (ASIC) capable of multiple samples on the rising edge of the fast pulse from an MCPPMT [4, 6], and intrinsic intra-chip jitter measured in picoseconds. In this case we have developed the PSEC4 ASIC, operated at 10.24 Gigasamples-per-second, with an effective dynamic range of 10.5 bits [10].

The OTPC prototype whose design and performance is described below differs from a future large detector in a number of ways. In particular, the small optical path length to the photodetectors allows ignoring dispersion of the photon velocities and scattering in the water, effects that can severely complicate exploiting precise timing for track reconstruction. While a full discussion is beyond the scope of the present paper, there exist strategies to ameliorate the effects: a) placement of the photodetectors (and potentially mirrors) on the detector surface and inside the volume such that there is always sufficient light with short path-length to seed the initial track hypothesis [11; b) restricting the wavelength range to limit the dispersion, making up the resulting inefficiency by additional photocoverage; c) using statistical methods and the varying of light paths from multiple photodetectors to perform a constrained fit to both the track and the dispersion photon-by-photon [12, 13, 14]; and, d), if and when panels of economical large-area LAPPD ${ }^{\mathrm{TM}}$ [15, 16] photodetectors become available, brute force photocathode coverage on the surface and throughout the volume of the detector, for example.

\subsection{A prototype $O T P C$}

To demonstrate this technique, we have built a $40 \mathrm{~kg}$ water Cherenkov detector using a combination of commercial MCP-PMTs and optical mirrors. A concept drawing of the optical setup of one of the five MCP-PMT's of this detector is shown in Figure 1. For each planar MCP-PMT mounted on the water volume, a mirror is mounted on the opposing side creating an image of the Cherenkov light hitting the opposing wall. For a given charged particle track, the mirror adds a discrete set of reflected photons that 


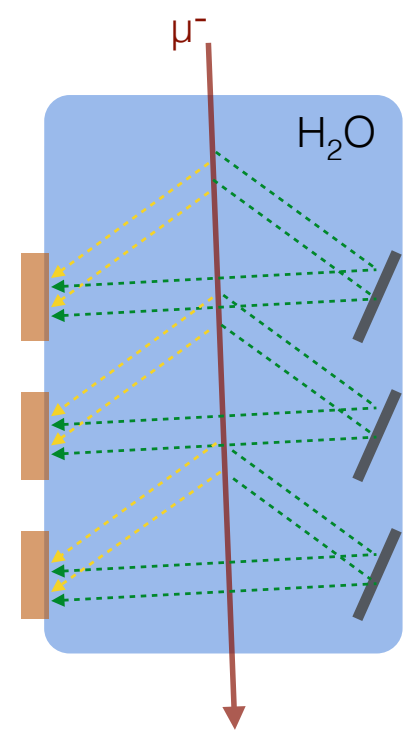

Figure 1: The concept of the prototype water-based OTPC. A charged particle emits Cherenkov light in the water volume. For each time and spatial-resolving MCP-PMT (left), a mirror is mounted on the opposing side. The mirror adds a discrete set of reflected photons (green) that arrive at the MCP-PMT after a delay due to the extra path length, and can be time-resolved from the direct photons (yellow).

can be time-resolved from the direct photons, thus economically increasing the photo-detection efficiency over the angular acceptance of the detector.

As the LAPPD MCP-PMTs are not yet available for use outside of a test setup [9, 17, this prototype relies on commercial MCP-PMTs that exhibit approximately the same detection resolutions, but with 1/16 the photo-active area per unit. The commercial photo-detector used is the Planacon XP85022 MCP-PMT device from PHOTONIS [18. The total photo-cathode coverage in the prototype OTPC is $125 \mathrm{~cm}^{2}$ of the surface area of $1700 \mathrm{~cm}^{2}$ that encloses the fiducial volume.

\subsection{Organization of the Paper}

The OTPC prototype detector is described in $\$ 2$, including the design of the optics in $\$ 2.1$ and details on the photodetector modules (PM) in $\$ 3$. The readout system, consisting of the fast waveform sampling digitizers of the front-end and the data-acquisition system (DAQ), is described in 8 . Section 5 describes the experimental setup in the Fermilab MCenter Test Beam area. The reduction of the raw data to times and positions is presented in $\$ 6$. Section 7 describes the response of the detector to single photons. The OTPC photo-detection efficiency and gain are discussed in Sections 8.1 and 8.2 . The test-beam results are shown in the final four sections, including the number of photo-electrons along the track $(\$ 9)$, resolving the direct and mirror-reflected Cherenkov photons and the time-projection along the beam axis $(\$ 10)$, the angular resolution $(\$ 11)$, and the spatial resolution of the reconstructed tracks $(\$ 12)$. The conclusions are given in $\$ 13$

\section{Detector}

The prototype OTPC detector is constructed from a $24 \mathrm{~cm}$ inner-diameter Poly-Vinyl Chloride (PVC) cylindrical pipe cut to a length of $77 \mathrm{~cm}$. Six $11 \mathrm{~cm}$ diameter ports were machined in the tube arranged 


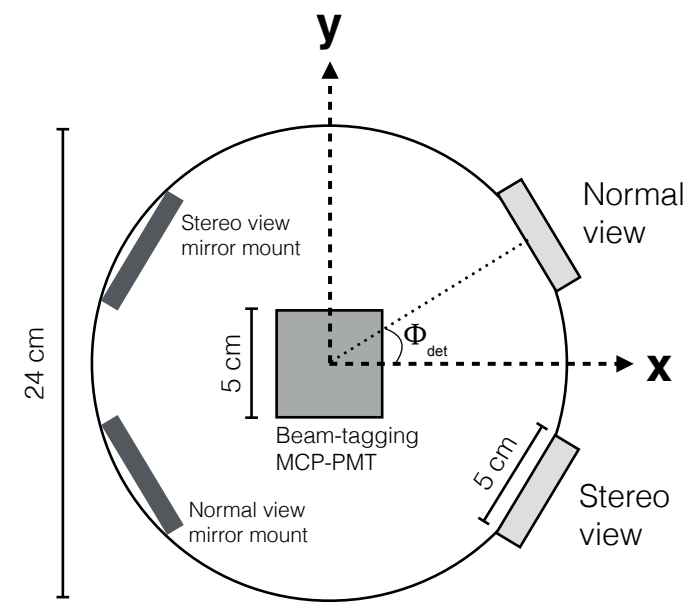

Figure 2: Rear-view scale drawing of OTPC detector showing the normal and stereo MCP-PMT mounting positions. The normal and stereo mounted PMs are symmetrical about the $\mathrm{x}$-axis in the OTPC coordinate system, and separated by an azimuthal angle of $\Phi_{d e t}=32.5^{\circ}$. The beam comes out of the page in the $+\mathrm{z}$ direction

in 2 columns of three ports each along the cylindrical axis, as shown in Figure 2 . These columns have an azimuthal separation of $65^{\circ}$. The photodetector modules (PMs), described in 3 , are mounted on five of these ports (the 6th port was occupied by a conventional PMT used as a diagnostic trigger). The column with 3 PMs installed is denoted the 'normal' view and the other the 'stereo' view.

For each PM, a first-surface broadband optical mirror is mounted on the opposing wall facing the PM port. The $7.6 \mathrm{~cm}$ square mirrors are installed on the interior wall of the cylinder at an angle of $24^{\circ}$, denoted as $\theta_{\text {mirror }}$ in Figure 3. The remaining exposed PVC surfaces of the interior wall are coated with light absorbing paint [19].

The detector is filled with $40 \mathrm{~L}$ of deionized water for the target volume. Because of the detector angular acceptance, the Cherenkov light directionality, and since only the first five ports are instrumented, the effective fiducial volume is limited to roughly the top two-thirds of the total volume, corresponding to a fiducial mass of $\sim 25 \mathrm{~kg}$.

\subsection{Detector Optics}

The optical properties of the detector components in the light path, as quoted by the manufacturers [18, 19], are shown versus wavelength in Fig. 4. Despite efforts to capture as much near-UV Cherenkov light as possible by using fused-silica windows, we found that these wavelengths are cut off by the optical coupling gel between the window of the port and the MCP-PMT. The characteristics of the light-absorbing paint were modeled with a broadband $95 \%$ absorbance and a $5 \%$ diffuse reflectance. A full detector simulation was built using the Chroma toolkit [20.

With MCP-PMT photodetectors having time resolutions of 100 psec or less and high spatial granularity, the water chromaticity and scattering properties become important as the distance from the particle track to the photo-detector increases. The Cherenkov photons, which are generated at an angle specified by the phase velocity of the dielectric medium, propagate at the group velocity, the speed at which energy is 


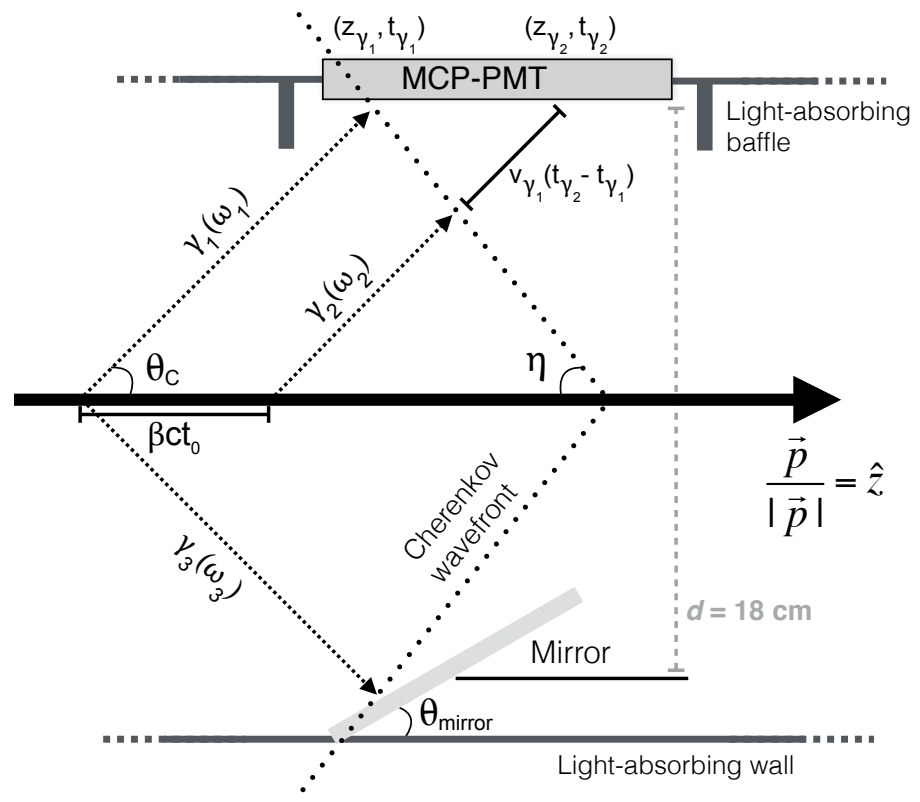

Figure 3: Two-dimensional projection of the Cherenkov light generated by a relativistic charged particle (heavy, black arrow) and the OTPC optics. The drawing shows the optical paths of three photons: $\gamma_{1}$ and $\gamma_{2}$ are directly detected, $\gamma_{3}$ is reflected at the mirror. The mirror is mounted at an angle, $\theta_{\text {mirror }}$, of $24^{\circ}$.

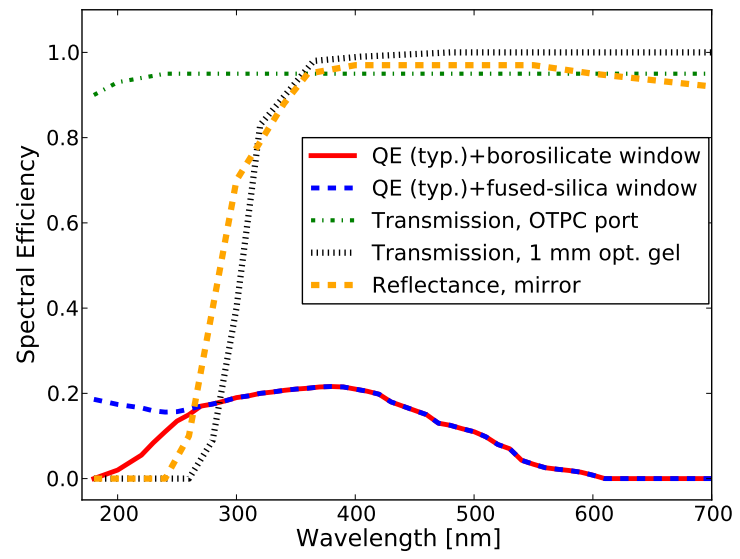

Figure 4: Optical properties of the OTPC optical components over wavelength range of 200 to $700 \mathrm{~nm}$. These parameterizations are used to model the optical properties in a simulation of the detector using the Chroma toolkit [20]. 


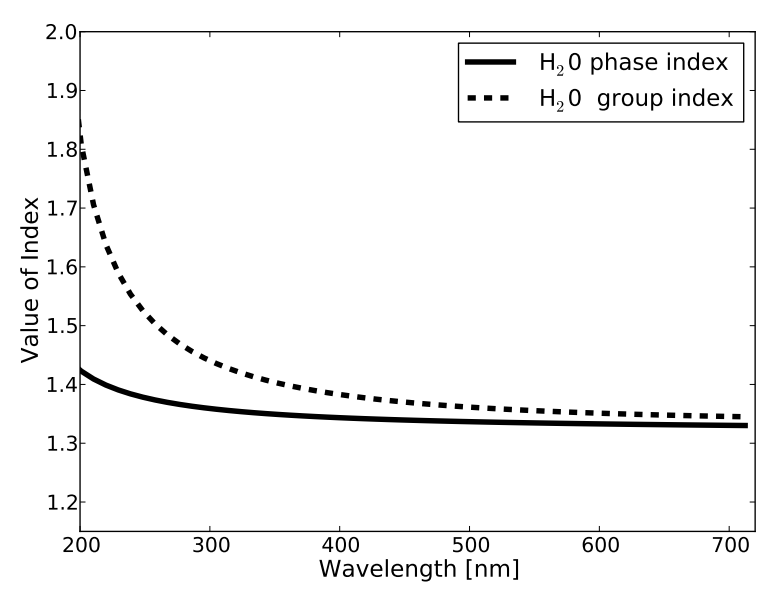

(a)

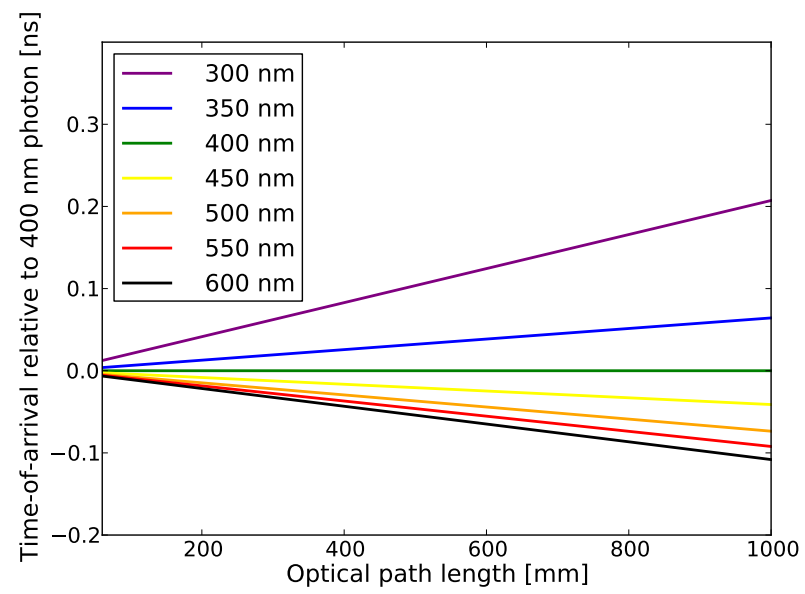

(b)

Figure 5: (a) Phase and group indices of refraction in water over the optical wavelength range. The water phase-index of refraction data were taken from [23. (b) Timing errors due to chromatic dispersion of the group velocity, referenced to a $400 \mathrm{~nm}$ photon, versus optical path length.

transported in the dielectric, given by [21]:

$$
v_{g}(\omega)=\frac{c}{n_{g}(\omega)}=\frac{c}{n(\omega)+\omega(d n / d \omega)}
$$

where $n(\omega)$ and $n_{g}(\omega)$ are the phase and group index of the medium, respectively. The group index is both greater in magnitude and more dispersive than $n(\omega)$ as shown in Figure 5 .

The spectrum of detected photons is the convolution of the OTPC spectral response and the Cherenkov spectrum 22. A simulation of $10^{4}$ muons with a uniform distribution of energies between 1 and $10 \mathrm{GeV}$ gives a spectrum of detected photons of $370_{-50}^{+110} \mathrm{~nm}$, with the spread covering about $95 \%$ of the spectrum around the mean wavelength. Over this wavelength range, $\left\langle n / n_{g}\right\rangle \approx 0.97$.

The relative timing errors due to chromatic dispersion of the group velocity are shown in Figure $5 \mathrm{p}$. At the maximum photon drift-length in the prototype OTPC $(\approx 30 \mathrm{~cm})$, the expected timing error is about $\pm 25 \mathrm{ps}$, which is small compared to the 75 ps single-photon timing resolution of the OTPC system shown in \$7. Averaging over the dispersion effects, the effective photon drift-speed in the OTPC is given by the weighted average of the group velocity over the detected photon spectrum, $<v_{\text {group }}>=218 \mathrm{~mm} \mathrm{~ns}^{-1}$.

Chromatic errors on the Cherenkov angle are also considered. For particles with $\beta=1, \theta_{C}=0.743_{-0.007}^{+0.008} \mathrm{rad}$ over $95 \%$ of the detected photon spectrum. For the longest photon path lengths of $\sim 30 \mathrm{~cm}$, this introduces maximum spatial (time) errors of $2.4 \mathrm{~mm}(\sim 10 \mathrm{ps})$, which are comparable or smaller than the photon detection resolutions in the prototype OTPC. The direct (non-reflected) photons travel less than $15 \mathrm{~cm}$, correspondingly reducing dispersion effects.

\subsection{Track reconstruction equations}

In the limit of negligible dispersion of the Cherenkov light, an analytic approach can be taken to reconstruct particle tracks. Consider a particle traveling through the OTPC as shown in Figure 3 , but further generalizing the track by allowing a polar angle, $\theta_{i}$, with respect to the z-axis (see the caption of Figure 2 ). 
Two Cherenkov photons generated along the particle path at $t=0$ and $t=t_{0}$ are detected with a relative time and longitudinal position of $\Delta t_{\gamma_{21}}=t_{\gamma_{2}}-t_{\gamma_{1}}$ and $\Delta z_{\gamma_{21}}=z_{\gamma_{2}}-z_{\gamma_{1}}$. The relative timing of the direct photons is given by

$$
\Delta t_{\gamma_{21}}=t_{o}+\left(\frac{L_{\gamma_{2}}}{v_{\gamma_{2}}}-\frac{L_{\gamma_{1}}}{v_{\gamma_{1}}}\right)=t_{o}+\frac{\Delta L_{\gamma_{21}}}{<v_{\text {group }}>}
$$

where $\Delta L_{\gamma_{21}}$ is the path length difference and $\left\langle v_{\text {group }}\right\rangle$ is the weighted average of the group velocity over the optical range of the detector. The experimentally measured relative times and z-positions in terms of the track variables are

$$
\Delta t_{\gamma_{21}}=t_{o}\left(1-\frac{\beta c}{<v_{\text {group }}>} \tan \theta_{i}\right)
$$

$$
\Delta z_{\gamma_{21}}=\beta c t_{o} \cos \theta_{i}
$$

A useful relation is the time-projection on the $\mathrm{z}$ coordinates. The projection over an infinitesimal track length is

$$
\frac{d t}{d z} \approx \frac{1}{\beta c}-\frac{\tan \theta_{i}}{<v_{\text {group }}>}
$$

for small angles along a path parallel to the detector plane. The OTPC photon drift speed in Eq. 4 is given by $<v_{\text {group }}>$.

An additional set of reflected photons is detected at each MCP-PMT from a mirror mounted on the opposite side of the inner cylinder as shown by $\gamma_{3}$ in Figure 3. This is not a necessary ingredient of an OTPC, but is an effective and economical method of increasing limited photocathode coverage. Each mirror is mounted at an angle, $\theta_{\text {mirror }}$ of $\left(90^{\circ}-\theta_{c}\right) / 2 \simeq 24^{\circ}$. For a small range of particle angles, the mirror creates a time-delayed image of the Cherenkov light on the opposing wall at the MCP-PMT.

The discrete mirror positions provide a technique to measure the longitudinal track location across a single PM by using the measured time difference between the direct and mirror-reflected Cherenkov light. For particle trajectories with small angles along the OTPC axis, the time-difference between a direct and mirror-reflected photon measured at the same z-position on the MCP-PMT is

$$
\Delta t(z)=\frac{1}{\left\langle v_{\text {group }}>\right.}\left(\frac{2 r}{\sin \theta_{c}}+d(z)\right)-\frac{1}{\beta c}\left(\frac{2 r}{\tan \theta_{c}}\right)
$$

where $r$ is the track displacement from the OTPC center-line and $d(z)$ is the inner cylinder diameter along the z-axis between the angled mirror and the MCP-PMT photocathode. The first term in Eq. 5 is the time separation from the path-length difference between the direct and mirror-reflected photons, whereas the second term accounts for the time-difference at which the photons were emitted along the particle track in order to arrive at the same z-position at the PM. Averaging over the dispersion effects and using $\beta=1$, we insert values in Eq. 5 and solve for the track displacement:

$$
r=0.737\left(\Delta t(z)<v_{\text {group }}>-d(z)\right)
$$

The scaling factor accounts for relative optical path lengths when the particle is off the OTPC centerline. The mean value of $d(z)$ is $18 \mathrm{~cm}$, which is the distance from the angled mirror center-point to the photo-detector module as shown in Figure 3. 


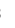

\section{Photodetector Module}

The OTPC is instrumented with five Photodetector Modules (PM), which provide the photon detection using the commercial 'Planacon' XP85022 MCP-PMT device from PHOTONIS [18. These square devices have a $5 \times 5 \mathrm{~cm}^{2}$ photo-active area and an anode comprised of a $32 \times 32$ square pad array with a $1.6 \mathrm{~mm}$ pad pitch. The three MCP-PMTs mounted in the normal view are $25 \mu \mathrm{m}$ MCP-pore-size tubes with fused silica windows manufactured in 2014-15. On the stereo view are 2 MCP-PMTs; one from the same manufacturing run, and another considerably older MCP-PMT with a borosilicate window. The latter MCP-PMT exhibited lower gain and detection efficiency.

While reading out each of the Planacon's 1024 anodes might provide the best-case granularity and pile-up rejection per channel, it presents a challenge when instrumenting a detector with many such photo-detectors. Instead, we adopt a similar microstrip readout scheme as the first-generation glass package LAPPDs [8, 7]. A thirty-two channel microstrip anode PCB board was designed such that a column of 32 anode pads is mapped to a single $50 \Omega$ transmission line. Each microstrip line serves as both the DC return and the high fidelity signal path for $\frac{1}{32}$ of the MCP-PMT. The central 30 strips are digitized while the two edge strips are terminated at $50 \Omega$. The board-mounted photo-tube is shown in Figure 6 .

The accelerated electron shower from the MCP will induce an electromagnetic wave between the microstrip's signal and reference plane, which will propagate equally along both directions on the 1D microstrip. We use a pair of high-density multi-channel $50 \Omega$ coaxial cables to bring these waveforms off the board [24]. The readout strategy adopted for the OTPC is to digitize the waveforms on the microstrips at only one end, leaving the multi-channel cables at the other end unterminated. Thus, the wave that initially propagates along a transmission-line microstrip toward the open end will be reflected with the same polarity back towards the digitizer for that strip, which then captures the waveforms caused by the initial pulse in both its direct and reflected manifestations (see Fig. 6] and \$4).

For a single photo-electron signal, the digitizer will record two transient waveforms along the microstrip. The times of the direct and open-end reflected waves, $t_{1}$ and $t_{2}$ can be extracted from these transients, as diagrammed in Figure 6. Using the measured times we can solve for the photon longitudinal position, $x$, and time-of-arrival, $t_{o}$ :

$$
x=v_{\text {prop }} \frac{t_{2}-t_{1}}{2}-\frac{D+2 C_{1}}{2}
$$

$$
t_{0}=\frac{t_{2}+t_{1}}{2}-\frac{1}{v_{\text {prop }}}\left(D+C_{2}+C_{1}\right)
$$

where $D, C_{1}$, and $C_{2}$ are the microstrip lengths of the MCP-PMT footprint, the reflected wave cable length, and the direct wave cable length respectively. The wave speed along the microstrip is $v_{\text {prop }}$, and is derived in $\$ 7.1$. A $10 "(25.4 \mathrm{~cm})$ high density coaxial cable provides a delay for the reflected signal and an identical 6" $(15.2 \mathrm{~cm})$ cable connects the microstrip card to the waveform digitizer board [24].

\section{Front-end data acquisition}

The waveforms on the anode microstrip of each PM are digitized at 10.24 Gigasamples-per-second (GSPS) using the 'ACquisition and Digitization with pseC4' (ACDC) printed circuit card. This card uses 30 channels of the PSEC4 ASIC with an analog bandwidth $\geq 1 \mathrm{GHz}[10$. The signal input to the PSEC4 is AC-coupled and the on-chip signal voltage range is from 0.1-1.1 V. A pedestal voltage-level of $0.8 \mathrm{~V}$ is set for the 


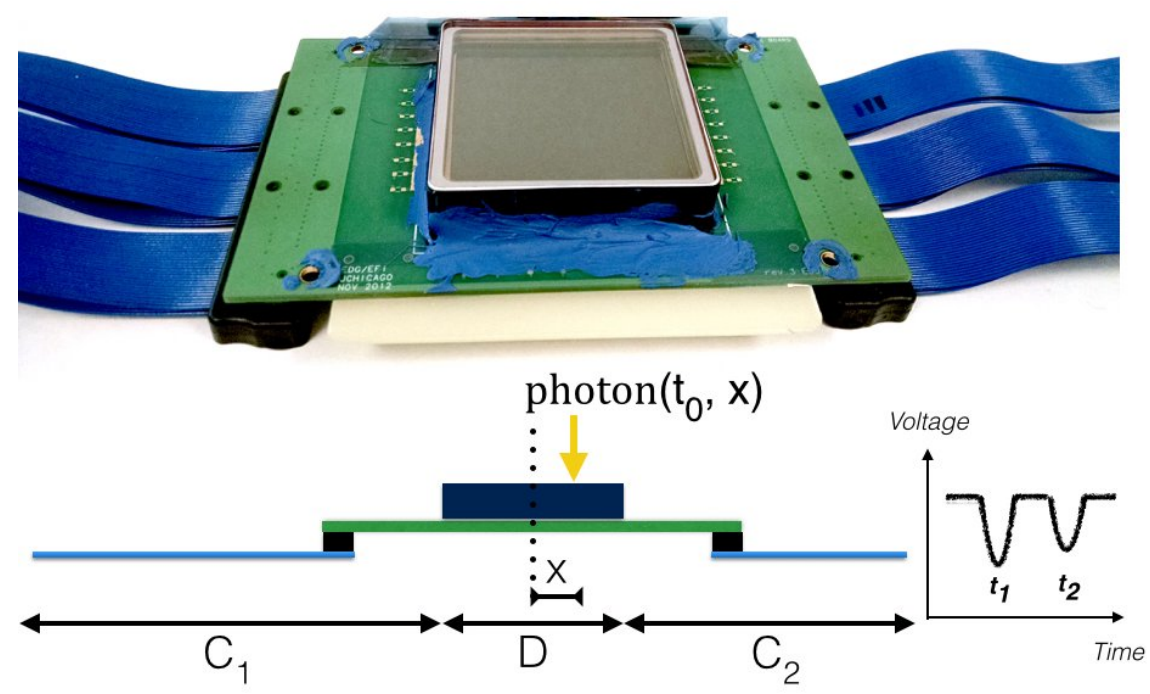

Figure 6: A PHOTONIS XP85022 Planacon MCP-PMT [18 mounted on a custom thirty-two channel, $50 \Omega$ transmission line anode board is shown in the top photograph. The Planacon has an anode of 1024 pads in a $32 \times 32$ array over the $5 \times 5 \mathrm{~cm}^{2}$ active area. These are mapped to the microstrip anode readout card in rows of 32. The bottom diagram outlines the technique for using the single-channel waveform timing to extract the incident photon position along the transmission line. The times of the direct and open-end reflected waves on the anode microstrip are denoted by $t_{1}$ and $t_{2}$.

OTPC data runs, allowing ample headroom for negative polarity pulses. The digitized waveforms from the PSEC4 chips are sent to an on-board field-programmable gate array (FPGA), which buffers the digital data. Serial communication and data readout to a system data acquisition card are performed over a low-voltage differential signaling (LVDS) cable link.

The ACDC card runs off a $40 \mathrm{MHz}$ clock that is up-converted within the PSEC4 ASIC using a voltagecontrolled delay line (VCDL) to achieve a $10.24 \mathrm{GSa} / \mathrm{s}$ sampling rate over 256 sample cells [10. Though an effective and power-efficient means of garnering high sampling rates, the VCDL-controlled switch capacitor array has non-uniform time-steps due to process variations in the integrated circuit fabrication $(\sim 10 \%$ spread in time-steps in PSEC4 [10). Methods of calibrating of the time-base of PSEC4 and related CMOS chips are discussed in [10, 25, 26. A complete, end-to-end PSEC4 time-base calibration was not performed on the data presented here, instead a first-order correction is applied such that the integral non-linearity over the time-base is zeroed. With this crude correction, a nominal time-step of 97 ps per PSEC4 sample is assigned.

\section{Experimental Setup}

The OTPC was installed on the beam line of the MCenter secondary beam at the Fermilab National Laboratory Test-Beam Facility [27, approximately $3 \mathrm{~m}$ behind a $1.09 \mathrm{~m}$ thick steel absorber. The absorber served as both a beam-stop for the secondary beam so that the dominant particle type seen by the OTPC was muons, and as a target station and collimator to generate a tertiary beam utilized by the LArIAT experiment, to which we operated parasitically 28 .

A side view of the installed OTPC is shown in Figure 7 where the upstream absorber is out of the frame. The five OTPC PM modules are mounted facing inwards on the water volume. Each of the PMs 


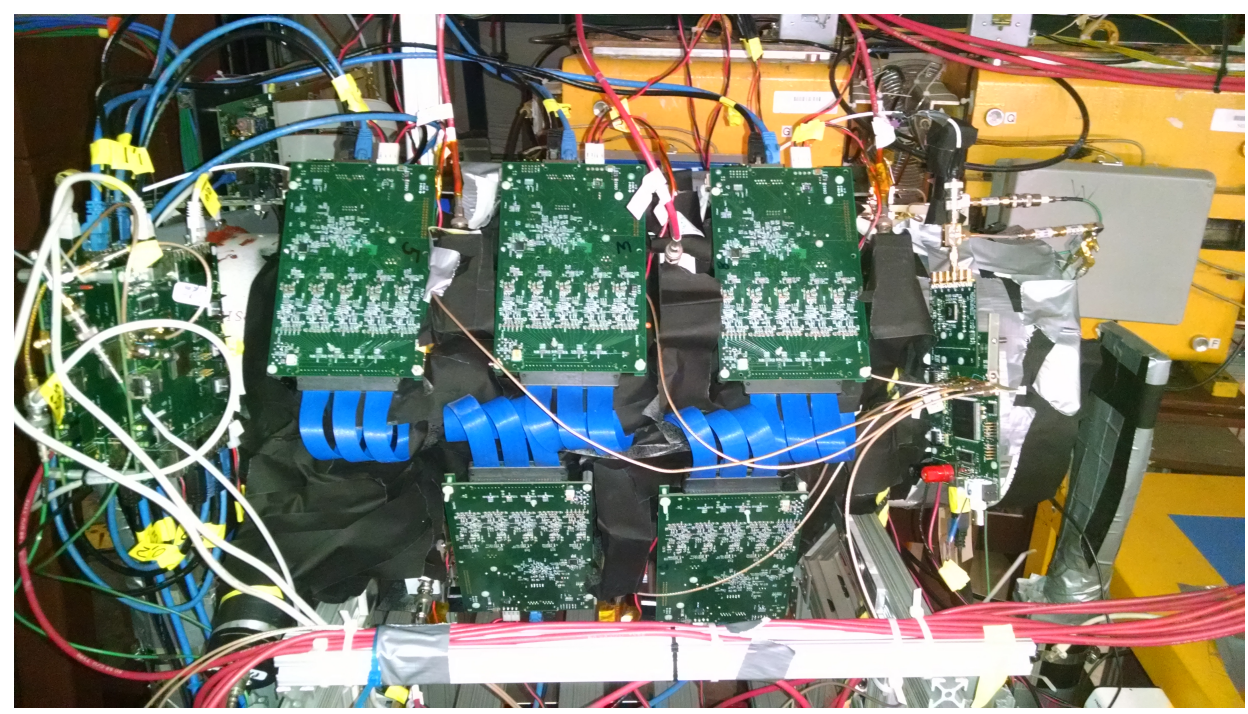

Figure 7: The prototype OTPC installed in the MCenter enclosure hall at the beam height of $208 \mathrm{~cm}$. The upstream steel absorber block is out of the frame to the right. Mounted facing inwards, the five PMs connect to the system data acquisition (DAQ) cards that are mounted on the left. The DAQ system connects to a remotely-controlled laptop on an adjacent rack, which also houses the beam trigger coincidence and discrimination logic. The yellow dipole magnets on the adjacent tertiary beam-line used by the LArIAT experiment are visible in the background.

is connected to the system central cards over two Category 6 (CAT6) network cables. The system cards interface to a remotely-controlled Linux PC on an adjacent rack that also houses the beam trigger coincidence and discrimination logic. High voltage (HV) is supplied to the detector from the counting room over $\sim 200 \mathrm{~m}$ SHV cables; the low voltage for the electronics is supplied within the enclosure. The OTPC water volume corresponds to 2.1 radiation lengths along the cylindrical axis.

The beam was delivered to the Meson Lab during the 4.2-second slow-spill at the beginning of the 60 second Fermilab accelerator complex 'super cycle'. During the OTPC test-beam runs, a secondary beam of positive pions impinged on the MCenter target at momenta of 8,16 , or $32 \mathrm{GeV} / \mathrm{c}$. Because the OTPC was located behind the secondary beam absorber, the particle flux was dominated by muons (85\%), with a mixture of hadrons $(10 \%)$, electrons $(1 \%)$, and photons $(4 \%)$ from punch-through and showering, as verified in a G4beamline simulation at a beam momentum of $16 \mathrm{GeV} / \mathrm{c}[29]$.

\subsection{The Beam Trigger}

The beam trigger consists of a pair of external scintillator detectors and a pair of Cherenkov detectors as shown in Figure 8. The pair of $5 \times 5 \mathrm{in}^{2}$ plastic scintillators mounted fore $\left(S_{1}\right)$ and aft $\left(S_{2}\right)$ of the OTPC provide good coverage over the OTPC cross-sectional area.

The two Cherenkov detectors $R_{1}$ and $R_{2}$, consist of MCP-PMTs with fused-silica Cherenkov radiators on their front faces that provide a prompt beam-trigger signal as the beam particle traverses the radiator and MCP-PMT. $R_{1}$, mounted on the front cap of the OTPC, consists of a PHOTONIS XP85011 MCP-PMT with an anode structure consisting of an 8-by-8 array of pixels, viewing a small cylindrical (1 cm diameter) fused-silica radiator mounted in its center. The $R_{1}$ signal is made by summing the central 32 pixels through $10 \Omega$ series resistors on each pixel, forming a single pixel of $1 \times 1 \mathrm{in}^{2}$ active area. The second Cherenkov 


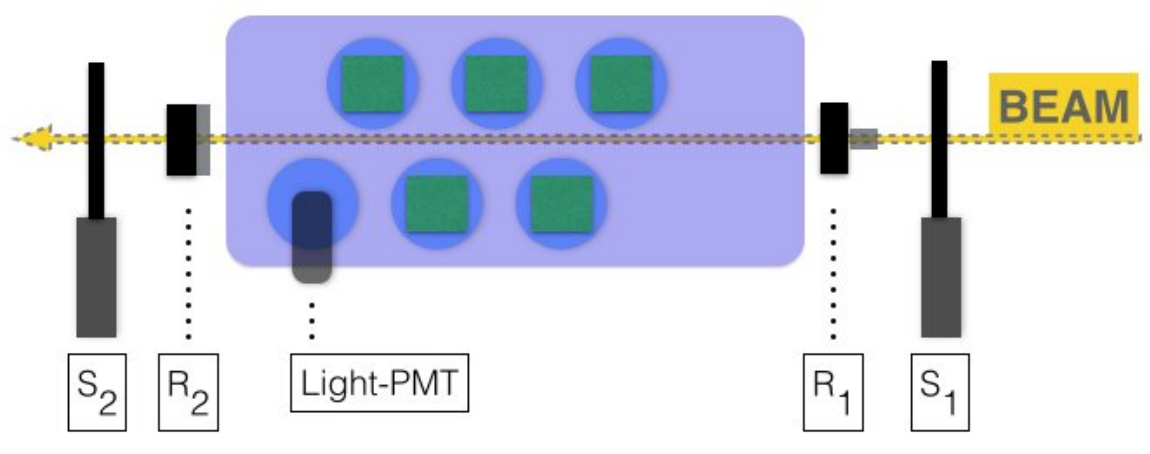

Figure 8: Diagram of the external beam-trigger (not to scale). The beam trigger coincidence can be made between four sub-detectors: the front and back scintillators+PMTs $\left(S_{1}\right.$ and $\left.S_{2}\right)$, and the front and back fused-silica Cherenkov radiators+MCP-PMTs $\left(R_{1}\right.$ and $\left.R_{2}\right)$. An additional diagnostic trigger is generated by a 1" PMT ('Light-PMT') mounted on the last OTPC port to observe Cherenkov light in the water volume.

counter, $R_{2}$, is mounted on the rear of the detector (see Figure 2), and consists of a PHOTONIS XP85022 MCP-PMT with an anode structure of 32-by-32 pixels read out by a 30-microstrip anode board, coupled to a matching $5 \mathrm{~mm}$ thick square fused-silica radiator.

Twenty-nine of the $30 R_{2}$ strips are read out and digitized with an ACDC board in the same manner as the PMs, allowing spatial and time-tagging of the out-going particle. The signal from $R_{1}$ is recorded in the 30th channel, which allows for a time-of-flight measurement for each through-going particle with approximately 100 ps resolution.

The beam trigger signal is made with standard NIM electronics. The signals $S_{1}, S_{2}$, and $R_{1}$ are discriminated and and fed to a coincidence unit. Two configurations were used during data runs: (1) $S_{1}+S_{2}+R_{1}$, and (2) $S_{1}+R_{1}$. Trigger configuration (1) is the default through-going particle trigger. Configuration (2) allowed the recording of particles that may scatter out, stop, or shower within the water volume. Both digital trigger signals are sent to the OTPC data acquisition system, which then passes these trigger signals to the front-end ACDC cards. For further details on the trigger system see [30].

\section{Data Reduction}

An OTPC event is recorded using 180 channels of 10.24 GSPS data in a time window of 25 ns. The first 150 channels contain the data from the OTPC photodetector modules (PM) and the remaining thirty channels, 150-178, contain the trigger information from the $R_{2}$ detectors (described in $\$ 5.1$ ). The digitized signal from $R_{1}$ (see Fig. 8) is saved in channel 179 and is used as a coarse zero-time reference.

The following procedures are performed on these data before analyzing an event:

1. A pedestal calibration is performed for each of the 256 sample cells in all PSEC4 channels 10 .

2. The firmware time-stamps are checked on the front-end ACDC boards. If the time-stamps are not synchronized, the event is discarded.

3. The Cherenkov data on all channels are located in the PSEC4 buffer using $R_{1}$ peak-signal time.

4. A reference baseline level, created using the median value of the 50 samples preceding the pulses, is subtracted from each channel. 

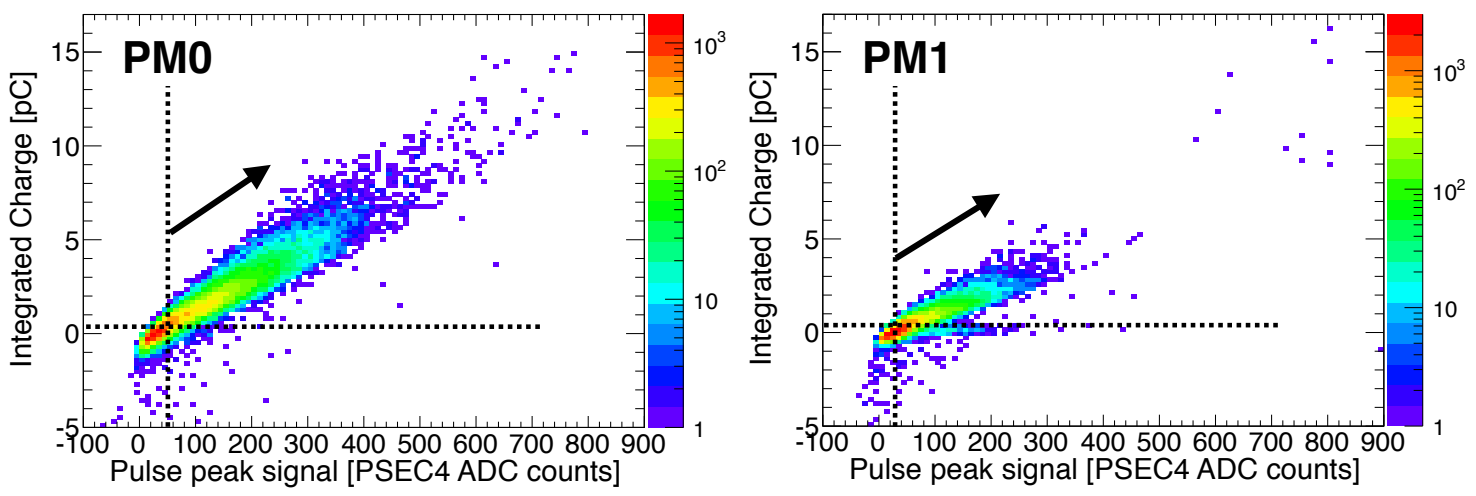

Figure 9: Correlation plots of integrated charge vs. pulse peak signal for PMs 0 and 1. Channels with waveforms above a minimum integrated charge and ADC count threshold, shown as dashed lines, are saved and further processed to measure the photon time-of-arrival.

5. A time-base is constructed from the PSEC4 samples after applying the time-base calibrations. The data points are cubic-spline interpolated to have an evenly spaced event time-base of 97 ps per sample.

At this point in the data reduction process, the event is aligned with respect to the $R_{1}$ trigger signal, baseline-subtracted, and referenced to a time-base. The event size is: 180 channels $\times 12$ bit ADC counts $\times$ 260 time-steps over $25 \mathrm{~ns}(70 \mathrm{kB})$.

The event is further reduced by extracting the trigger information in channels 150-179, narrowing the event time-window to hold only the region of Cherenkov photon hits, and removing below-threshold channels as shown in Figure 9 30. After the data reduction, the event comprises the following trigger information: particle output x-position, particle output y-position, time-of-flight, $R_{1}$ trigger time, $R_{2}$ trigger time, $R_{1}$ total charge, $R_{2}$ total charge, and the number of distinct hits in $R_{2}$. The reduced size of the event timestream data is: number of OTPC channels above threshold $(\leq 150) \times 12$ bit ADC counts $\times 110$ time-steps over $10.67 \mathrm{~ns}$ (number of channels $\times 0.17 \mathrm{kB}$ ).

\subsection{Extracting the photon time-of-arrival}

For each detected photon, we measure its $2 \mathrm{D}$ spatial position on the OTPC cylinder $\left(z_{\text {det }}, \phi_{\text {det }}\right)$ and its time-of-arrival, $t_{d e t}$. By projecting the measured photon arrival times on the two spatial coordinates we can reconstruct the charged particle track in 3D. We take the detected photon azimuth angle, $\phi_{\text {det }}$, to be the PM location about the OTPC cylinder and its z-position, $z_{\text {det }}$, as the microstrip position along the OTPC longitudinal axis. There are 2 discrete $\phi_{\text {det }}$ angles and 150 discrete $z_{\text {det }}$ positions. This section describes how we extract the time-of-arrival for each photon from the digitized waveforms.

We found that a robust algorithm is to interpolate the PSEC4 waveform in a small region around the peak. A window of 4 time-steps (97 ps each) is interpolated with a cubic spline on a 9.7 ps grid and the pulse time is taken from the peak of the interpolated region. An example of an OTPC channel hit by a Cherenkov photon is shown in Figure 10.

For an event, the output of the peak-interpolation algorithm is a set of times $\left(\left[t_{1 a}, ..\right],\left[t_{2 a}, \ldots\right]\right)$ from the direct and anode-reflected pulses. To get the photon hit time, we apply Eq. $7 \mathrm{~b}$ and require causality using Eq. 7a. That is, the time difference between the direct and anode-reflected pulse should be located on the 


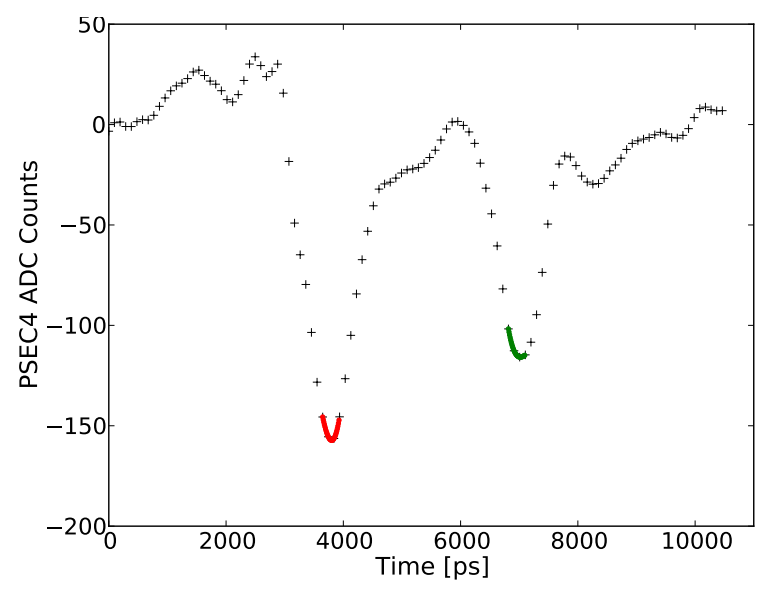

Figure 10: OTPC digitized waveform from a Cherenkov photon. A single photo-electron waveform is shown with the peak-interpolation time-extraction method. The interpolated peak-signal regions on the waveform are shown in red (green), which represent the direct (anode-reflected) pulses.

microstrip line within the $5 \mathrm{~cm}$ length of the MCP-PMT. The causality requirement is that $t_{2 a}-t_{1 a} \in[3.1$, 3.9] ns after solving Eq. $7 \mathrm{a}$ for the cable lengths in the PM.

\section{Single photo-electron response}

A laser test-stand was built to characterize the single photo-electron response of the PMs. A $405 \mathrm{~nm}$ PiLas laser was used in pulsed mode, which can achieve pulse widths of 33 ps FWHM 31 .

\subsection{Single-photon}

The single photo-electron position resolution is obtained by scanning the laser beam, attenuated to the single-photon level, on the active area of the PMs both parallel and transverse to the microstrip direction. Results from a scan along the microstrip direction are shown in Figure 11. Four data points were taken at a spatial separation of $4.5 \mathrm{~mm}$ with a time-difference resolution of $\sim 35 \mathrm{ps}$ measured for all laser positions. This is the inherent electronics timing resolution for the single photo-electron pulses digitized using one channel of the PSEC4 chip, with the basic (not complete) time-base calibration described in \$4

Figure 11 shows the time difference along the microstrip vs. the laser beam position, from which the signal propagation velocity, $v_{\text {prop }}$, in Eq. 7 along the microstrip to be calculated. The best fit $v_{\text {prop }}$ is found to be $0.47 \pm 0.02 c$.

\subsection{Multi-photon}

A similar test is employed to measure the relative timing between single photons within the same laser pulse. In this setting, an iris is aligned with the laser beam axis and a $10 \mathrm{~mm}$ focal length convex lens is put in the laser path. The MCP-PMT sits $30 \mathrm{~cm}$ behind the lens, which projects an elliptical laser beam spot over the MCP-PMT active area. A neutral density filter is installed after the lens to attenuate to an average of a few photons per pulse.

Events are analyzed that have two recorded photo-electrons, in which the signals are clearly separated between channels above and below the MCP-PMT center-line. The relative timing between photons is 


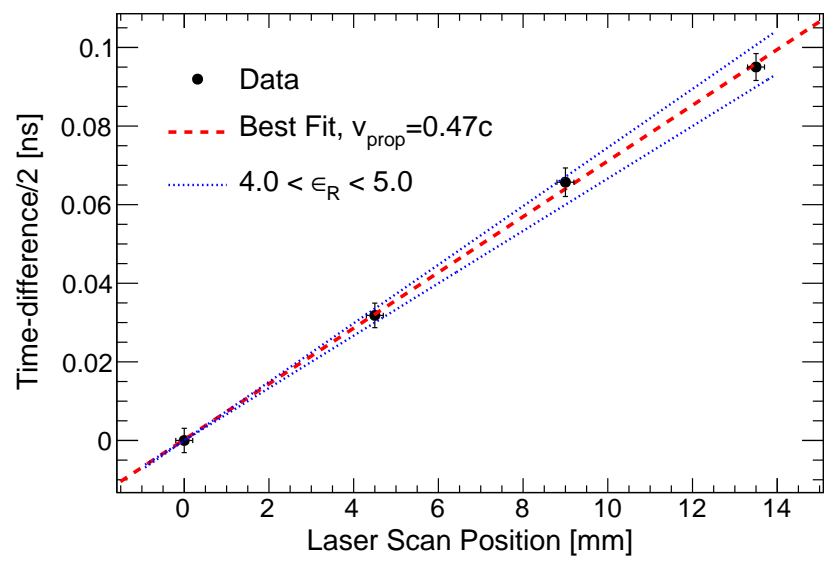

Figure 11: Single photo-electron laser scan to measure the propagation velocity along the microstrip line. The vertical error bars are the $3 \sigma$ standard error on the time-difference measurement (the single event statistical error on the time-difference is $\leq 35 \mathrm{ps}$ for all laser positions). The data agree with the expected velocity given the typical dielectric permittivity of an FR4 printed-circuit board substrate $(\epsilon=4.3 \pm 0.2)$.

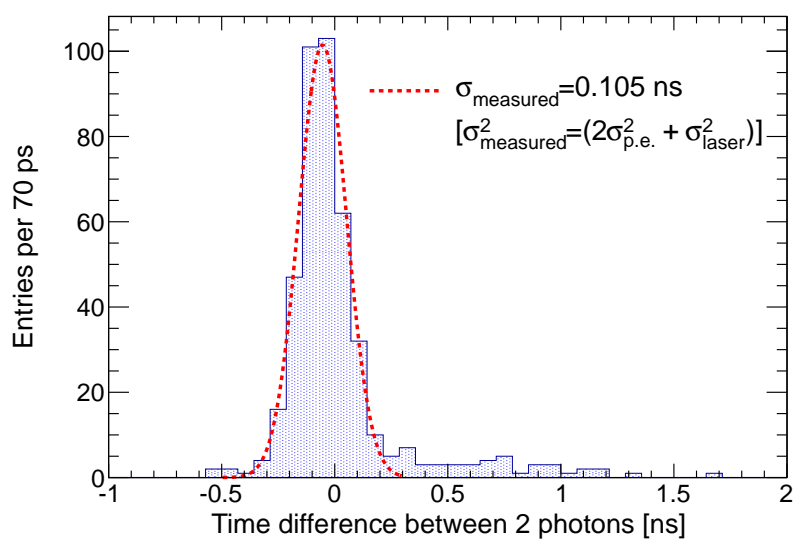

Figure 12: Relative timing measurement between 2 photons on the PM. The underlying single photo-electron electronics + MCP-PMT timing resolution for this measurement is 75 picoseconds.

shown in Figure 12, A tail is seen in the distribution, which is likely caused by optical reflections in the test setup that are recorded late. The core resolution is shown to be $105 \mathrm{ps}$, which corresponds to a single photo-electron PM resolution of 75 ps.

From these measurements, we conclude that the 1- $\sigma$ single photo-electron time-of-arrival and space statistical errors are $\left(\sigma_{t}, \sigma_{x}, \sigma_{y}\right)=(75 \mathrm{ps}, 2 \mathrm{~mm}, 3 \mathrm{~mm})$. The $\sigma_{x}$ resolution is taken from the microstrip pitch on the anode board.

\section{Photo-detection properties}

Photo-detection properties were derived from a dataset of 1686 events taken in $16 \mathrm{GeV} / \mathrm{c}$ secondary beam runs using the beam-trigger configuration 1 as described in \$5.1). 


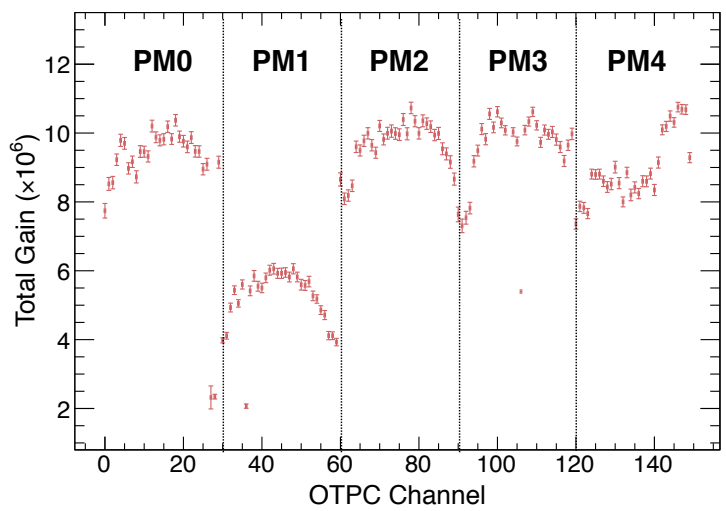

Figure 13: The OTPC per-channel gain. PM 0, 2, and 4 constitute the OTPC normal view; PM 1 and 3 are in the stereo view. The gain factor on each channel is comprised of three factors: the MCP-PMT $\times$ electronics pre-amp gain $\times$ variations in the PSEC4 count-to-voltage transfer function.

\subsection{Photo-detection efficiency}

A comparison between data and the expectation from simulation shows a $70-80 \%$ detection efficiency compared to our detector Monte Carlo, which assumes optimal coupling and equal photo-response at each PM. We estimate that the losses of the direct Cherenkov light are due to: (1) $10 \pm 5 \%$ due to reflection losses (spots poor optical-gel coupling would lead to total-internal reflection), and (2) $6 \pm 3 \%$ due to light cut off by a baffle around the photodetectors. Additional light collection inefficiencies (for both direct and reflected light) are: (3) $5 \pm 1 \%$ from the lower efficiency of PM1; and (4) $3 \pm 1 \%$ from 5 readout channels with poor coupling, which were removed from the analysis chain. These estimates are consistent with the overall observed detection efficiency, and the measurements of the lower number of direct photons relative to reflected photons shown in $\$ 10.1$.

\subsection{Single photo-electron gain}

The gain per channel is shown in Figure 13, which is the multiplicative gain of the MCP-PMT, the $20 \mathrm{~dB}$ pre-amplifier board, and any relative gains between channels in the PSEC4 ASIC ${ }^{1}$. This is measured by taking the median integrated charge for signals above threshold over the dataset. Error bars are taken to be the RMS of the integrated charge distribution per channel. MCP-PMT gains of about $10^{6}$ are found for PMs 0 and 2-4, who are measured to be about twice the gain of PM 1. The gain on each channel is used to calibrate the number of detected photo-electrons per event.

\section{Photo-electrons along the track}

Four raw time-projected events are shown in Figure 14. The PSEC4 time-step is plotted versus the OTPC channel, which is divided into 5 sub-panels representing each PM. The raw PSEC4 signal amplitudes

\footnotetext{
${ }^{1}$ Since a full voltage calibration was not performed on these data, there exist small gain differences between PSEC4 channels due to their relative transfer functions from input voltage to output ADC counts. The vast majority of signals are small $(<10 \%$ of the PSEC4 voltage range [10], so this is not a large effect.
} 


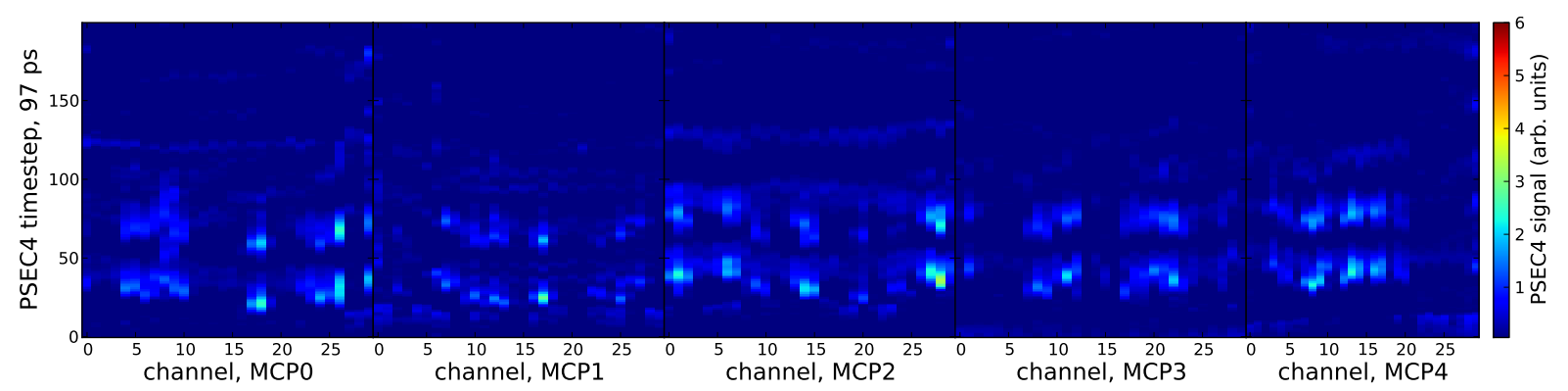

(a)

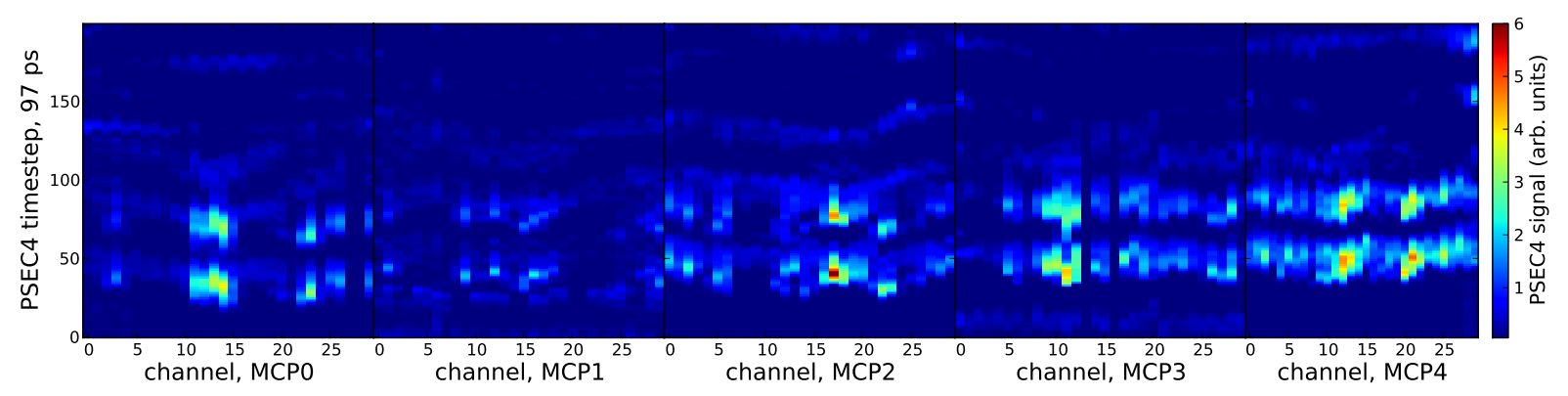

(b)

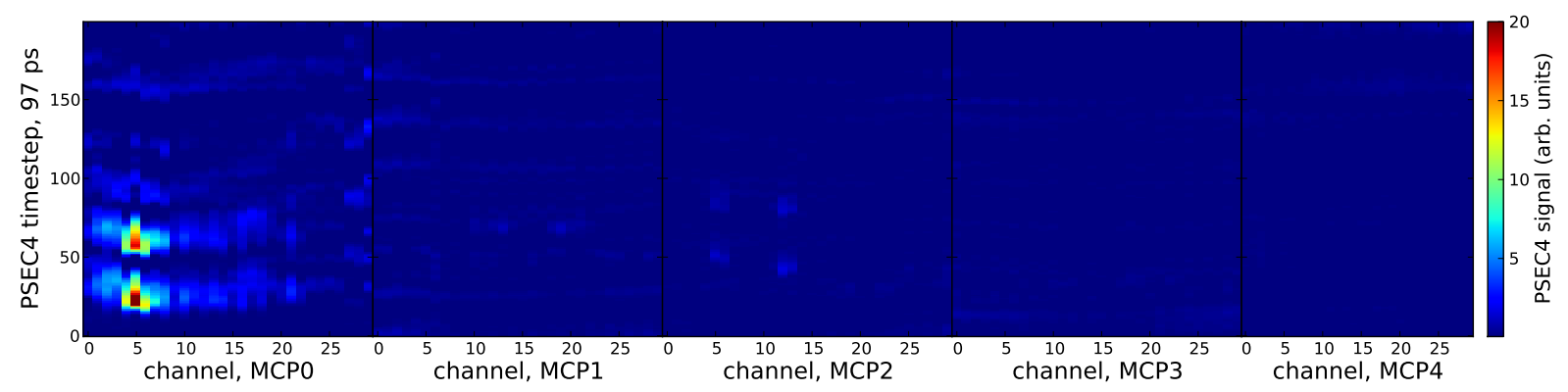

(c)

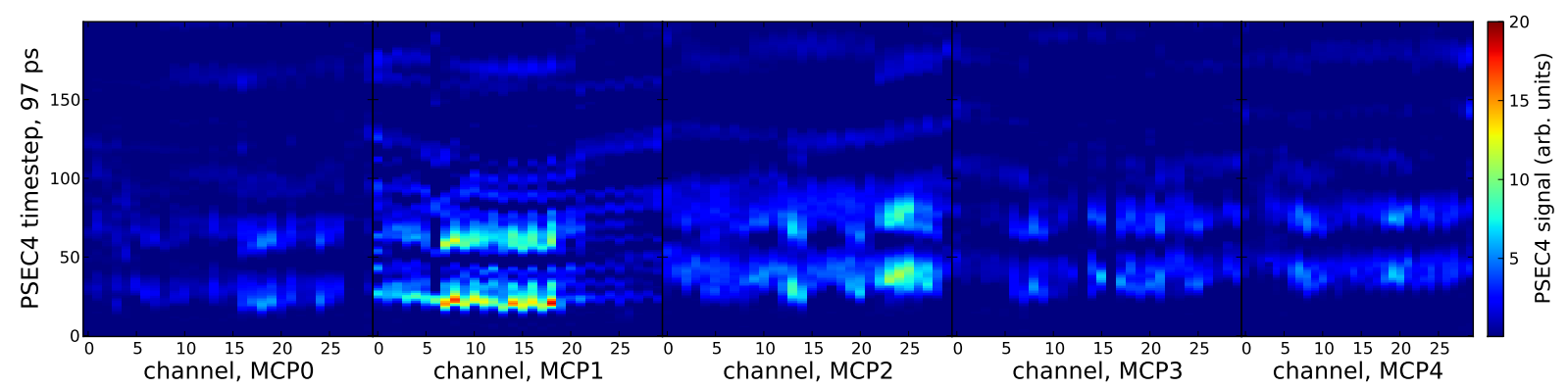

(d)

Figure 14: Raw OTPC events: time-step (97 ps per bin) vs. OTPC channel. The 5 panels in each event are the individual OTPC PMs. PMs 0, 2, and 4 constitute the OTPC normal view, PMs 1 and 3 are in the stereo view. The raw PSEC4 signal amplitudes are normalized with respect to the average gain on each PM. (a) Typical through-going event that is representative of most recorded events. (b) Through-going event, in which the number of detected photons increases near the right side. (c) Track with a large number of photons over a short extent. (d) Large signal amplitude along entire track, peaking in the 2nd panel. Events (c) and (d) were recorded using trigger configuration 2 (5.1). Events (a) and (b) were recorded using configuration 1 , the through-going trigger mode. 


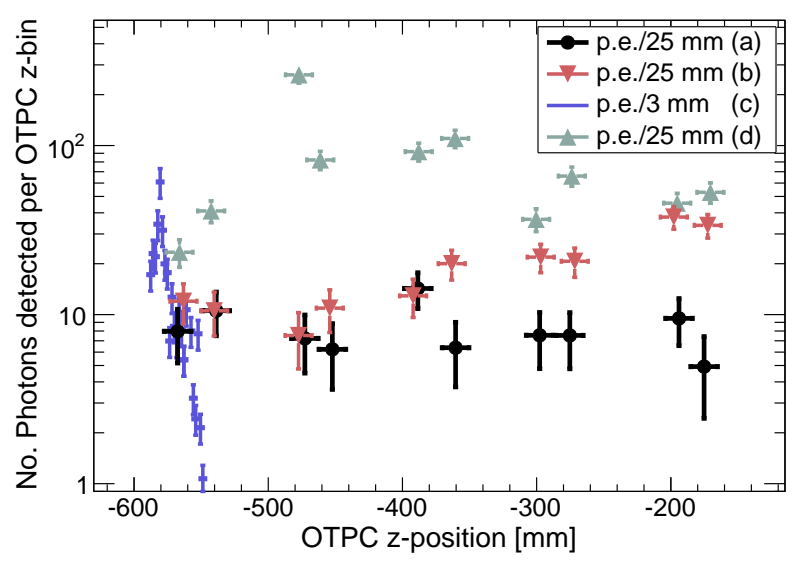

Figure 15: The number of photons detected along the OTPC z-axis, per z-bin defined by the horizontal error bars, for events presented in Fig. 14 The gain calibrations shown in 88.2 were used to convert the waveform integrated charge to a number of photo-electrons (p.e.). Events a, b, and d show the number of p.e.'s per $2.5 \mathrm{~cm}$ interval along the OTPC z-axis. Event c shows the number of p.e.'s per $3 \mathrm{~mm}$.

are normalized with respect to the average gain on each PM. The beam direction is from left-to-right. The two time-separated pulses for every signal channel are the direct and anode-reflected waveforms. These data are reduced up to step 5 in 6 .

Events (a) and (b) in Fig. 14 are from the $16 \mathrm{GeV} / \mathrm{c}$ data that were recorded using the through-going trigger configuration. Events (c) and (d) are from the $8 \mathrm{GeV} / \mathrm{c}$ data, which were recorded using trigger configuration 2. These two tracks left no signal in $R_{2}$, implying that these particles interacted in the volume, or else somehow missed the back trigger detector.

By applying the channel-to-channel gain calibration described in $\$ 8.2$, we can measure the effective number of photo-electrons along the track. Figure 15 shows the number of detected photons along the OTPC z-axis for the events in Fig. 14. Event (a) has a roughly uniform number of photo-electrons along the $\sim 40 \mathrm{~cm}$ extent of the photo-detection coverage. Event (b) shows an increase in the detected photons in the last one-third of the track, possibly due to the creation of a $\delta$-ray along the track. Events (c) and (d) are distinct in that they exhibit a large localized peak in the number of detected photons, suggesting that these events may have an EM showering component. Event (c) could also be explained by an wide-angled muon track, either from the beam or an accidental cosmic ray, that exited the OTPC volume at PM 0.

\section{The time-projection}

In this and the following sections we use 1230 events from a secondary beam momentum of $16 \mathrm{GeV} / \mathrm{c}$ dataset, which were triggered on through-going tracks at the best measured water quality.

We now add the time dimension of the detected photons, as extracted from the waveforms in 86.1 , to the data analysis. Figure 16 shows the measured time structure of the events, in which the times-of-arrival of the Cherenkov photons on the OTPC normal view are plotted with the measured times of the beam trigger signals, $R_{1}$ and $R_{2}$. The time structure seen in the Cherenkov photon data is due to the three discrete PM locations in the OTPC normal view. 


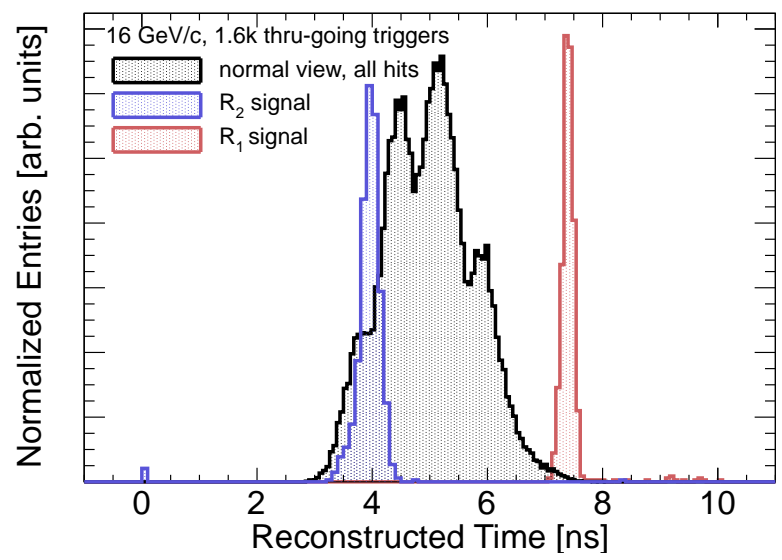

Figure 16: The times of the data and trigger signals from the reduced $16 \mathrm{GeV} / \mathrm{c}$ dataset. The reconstructed times of the detected Cherenkov photons (from the normal-view) are shown by the black histogram. The 'fast' trigger signals, $R_{1}$ and $R_{2}$ are shown in the red and blue, respectively. Each event is aligned with respect to the $R_{1}$ time. The time of $R_{1}$ is measured later than the $R_{2}$ signal due to cable delays.

\subsection{Resolving the direct and mirror-reflected Cherenkov photons}

As shown in Equation 4 , the particle's time-projection along the longitudinal axis has two components: one from the particle's velocity and the other from its angle with respect to the OTPC z-axis. For throughgoing multi-GeV muons, we can assume the particle's velocity is constant along the OTPC with $\beta=1$. We introduce the position-corrected time, $t^{\prime}$, which nulls out the velocity term in Eq. 4

$$
t_{i}^{\prime}=t_{i}-\frac{z_{i}}{c}
$$

$$
\frac{d t^{\prime}}{d z}=\frac{d t}{d z}-\frac{1}{c}=\frac{\tan \theta}{<v_{\text {group }}>}
$$

where $t_{i}$ and $z_{i}$ are the measured individual photon times and z-positions. As defined in $\$ 2.1$, $<v_{\text {group }}>$ is the weighted average of the group velocity dispersion over the optical efficiency range of the detector. In the $t^{\prime}$ basis, the time-projection along the OTPC z-axis is a measure of the particle angle.

Figure 17 shows the distribution of the measured photon times in terms of their position-corrected time, $t_{i}^{\prime}$. The $R_{2}$ trigger time for each track serves as a fine time-alignment between events. As expected, the $t^{\prime}$ distribution is bi-modal with peaks corresponding to the direct and mirror-reflected photons. A distinct separation of $770 \mathrm{ps}$ is observed, which is consistent with the path length differences between the direct and reflected photons divided by the photon drift velocity, $\left\langle v_{\text {group }}\right\rangle$. The path length difference is given by the range of distances between the angled mirror and PM photocathode, $18 \pm 1 \mathrm{~cm}(\$ 2.2)$, which corresponds to an expected time difference between the direct and mirror-reflected photons of $830 \pm 50$ ps.

Figure 17 also shows a larger number of mirror-reflected photons than direct photons. Using the relative areas from the Gaussian fits to the distributions, we measure fewer direct than reflected photons by a factor of 0.55 in the normal view data. This was unexpected; the detector Monte Carlo predicts approximately equal numbers of each for small-angle tracks. The relative deficit of direct photons is most likely due to reflections of the direct light at the fused-silica OTPC port and MCP-PMT interface as discussed in 98.1 It 


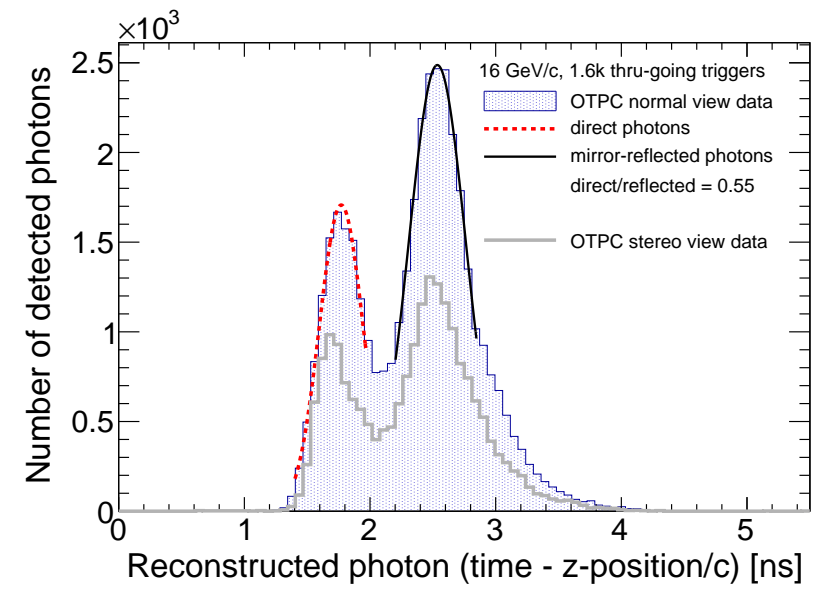

Figure 17: Histogram of the inclusive dataset of detected photons using the position-corrected time: $t_{i}^{\prime}=$ $t_{i}-z_{i} / c$. This removes the contribution to the time-projection along the z-axis due to the particle's velocity (assuming $\beta=1$ ). The direct and mirror-reflected Cherenkov photons are distinctly visible and separated by $770 \mathrm{ps}$, as taken from the fit parameters.

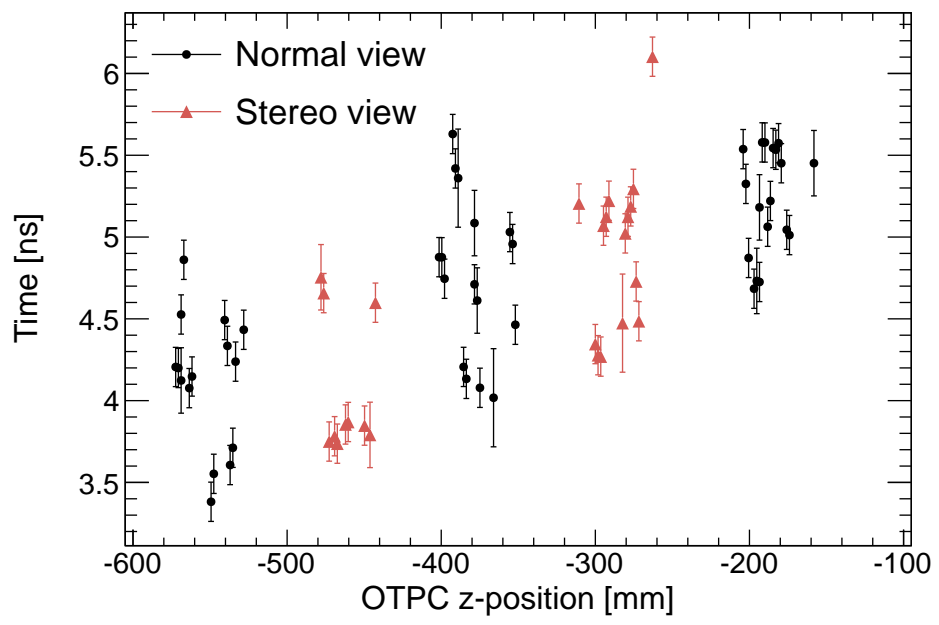

Figure 18: The time-projection along the beam axis for a single event. The time projection along the z-axis is shown for the event shown in Figure 14 asing 73 individually resolved Cherenkov photons.

is also likely that there is a small percentage of scattered photons in the data; these would arrive later than the direct photons and may be included in the mirror-reflected distribution and also cause the observed tail in the $t^{\prime}$ timing distribution after $3 \mathrm{~ns}$.

\subsection{An example event}

Figure 18 a shows the time-projection on the z-axis for the raw event shown in Fig. 14a. In this event, 73 individual photons were resolved in both time and space. Figure 18 shows their measured times, $t_{i}$, projected versus their detected positions, $z_{i}$. The photons detected in the normal and stereo views are denoted by black and red data-points, respectively. Figure 19 is a histogram of the position-corrected times, $t_{i}^{\prime}$, of this 


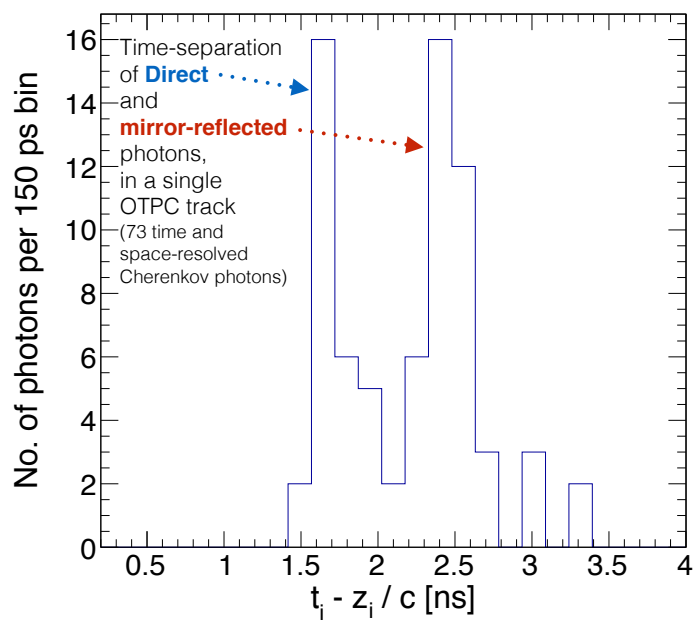

Figure 19: Histogram of the detected photon times from the event in Fig. 18, plotted using the positioncorrected time introduced in Fig. 17. The separated peaks in the time distribution are from the direct and mirror-reflected Cherenkov photons.

event. The peaks in the histogram are from the measured arrival-times of the direct and mirror-reflected photons.

\section{Angular resolution}

The direct Cherenkov photons can be used to measure the particle angle with respect to the OTPC z-axis as shown in Eq. 8. The most straightforward method of extracting this angle is to assume a linear track over the extent of the OTPC photodetector coverage. The slope of the linear fit, multiplied by the weighted average of the group velocity $\left.\left(<v_{\text {group }}\right\rangle\right)$, is the tangent of the track angle.

It is possible to separate the direct and mirror-reflected photons when using the position-corrected time, $t^{\prime}$, as shown in Figure 17. A normal view time-cut of $<1.95 \mathrm{~ns}$ and a stereo view time-cut of $<1.90$ ns were used on the data to isolate the direct photons. The number of direct Cherenkov photons per event is shown in Figure 20a. The sample fitted consists of 522 events with more than 8 normal-view and more than 4 stereo-view direct photons are fit, requiring that each PM has at least two direct photons. Separate linear fits to the direct stereo and normal view photons are performed. The fits are reasonable, as shown by the distributions of $\chi^{2}$ per degree of freedom in Figure $20 \mathrm{~b}$.

The returned slopes from the fits, $\frac{d t^{\prime}}{d z}$, are multiplied by $<v_{\text {group }}>=(\mathrm{c} / 1.38)$ and histogrammed in Figure 21. Linear fits to the photons in the direct normal-view yield an angle relative to the nominal beam axis over all the fitted tracks of $73 \pm 60 \mathrm{mrad}$ at $1 \sigma$ resolution. The stereo view shows a measurement of $-20 \pm 121 \mathrm{mrad}$. The stereo view measurement has a larger error that is most likely due to its smaller zcoverage than the normal view, which gives it a smaller lever arm on the angular measurement. The normal view has 3 equally spaced PMs over a z-range of $\sim 40 \mathrm{~cm}$, but the stereo view has $2 \mathrm{PMs}$ covering a z-range of only $20 \mathrm{~cm}$. 


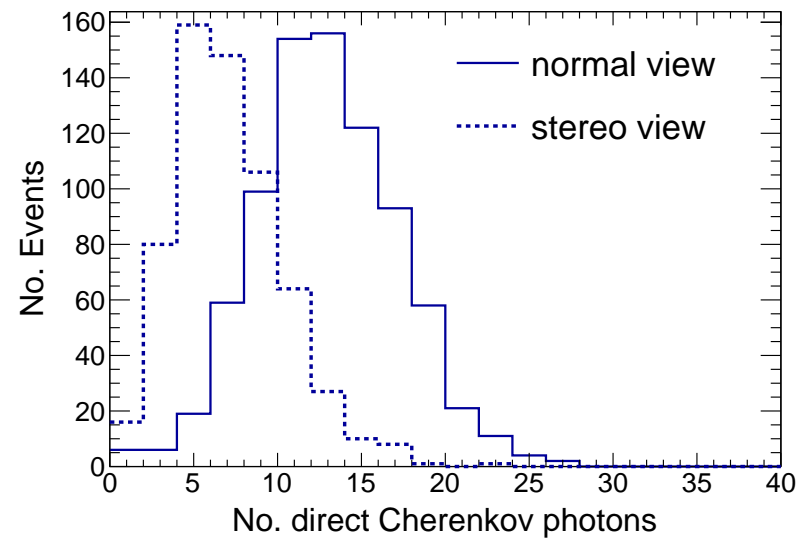

(a)

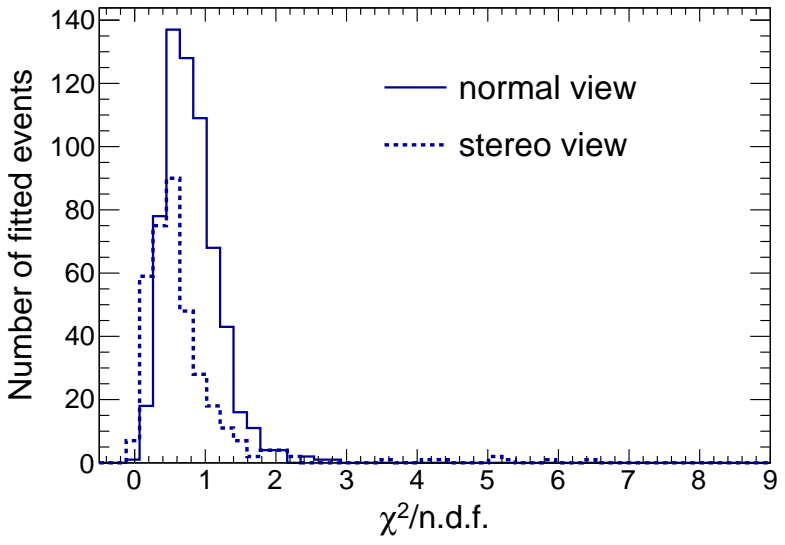

(b)

Figure 20: (a) Number of direct Cherenkov photons per event. The direct and mirror-reflected photons are separated by applying a time-cut to the data presented in Fig. 17. (b) Goodness of fit as given by the $\chi^{2}$ per number of degrees of freedom (n.d.f.). Events from (a) with more than 8 normal-view and more than 4 stereo-view direct photons are used in the fit.

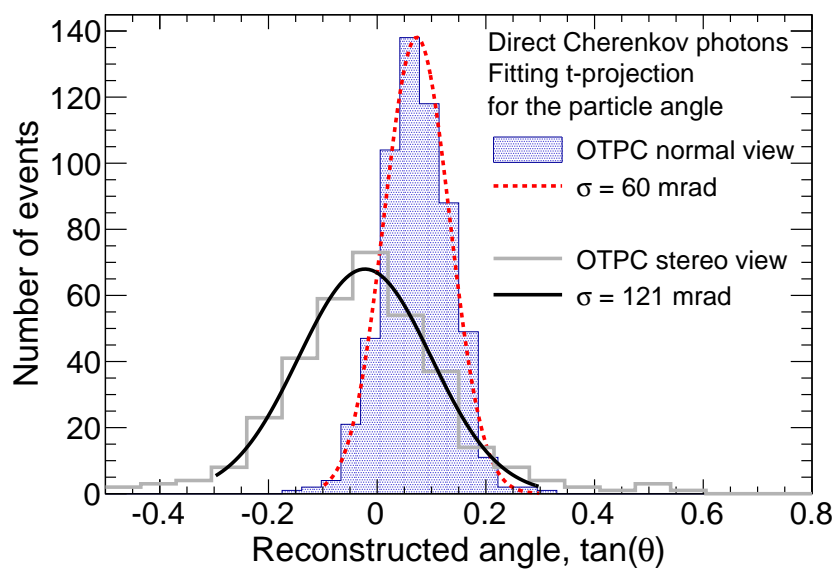

Figure 21: Measured angle of tracks along the z-axis as seen by the stereo and normal views. The direct Cherenkov light is fit with a straight line for events with more than 8 direct normal-view photons. In the stereo view, at least 4 photons are required to fit the angle. The measured angle distributions are: $73 \pm 60 \mathrm{mrad}$ for the normal view, $-20 \pm 121 \mathrm{mrad}$ in the stereo view. Fits that have a $\chi^{2} /$ n.d.f greater than 3, as shown in Fig. 20p, were not included in this measurement. 


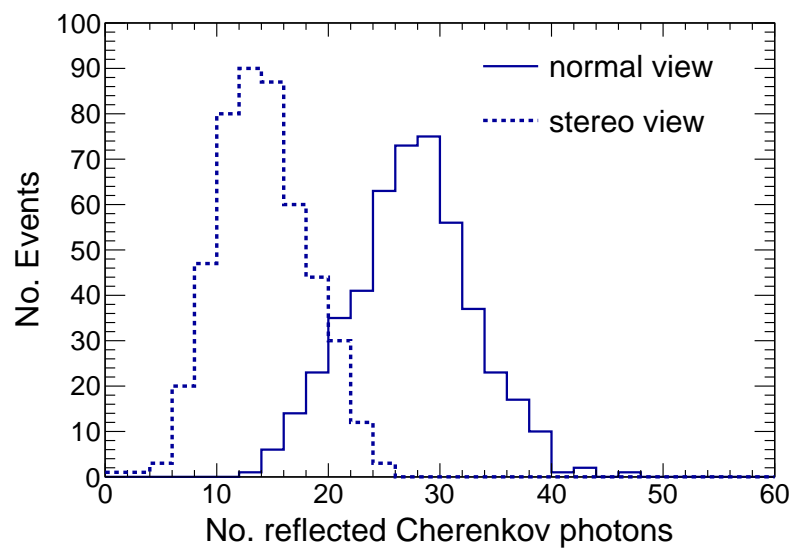

(a)

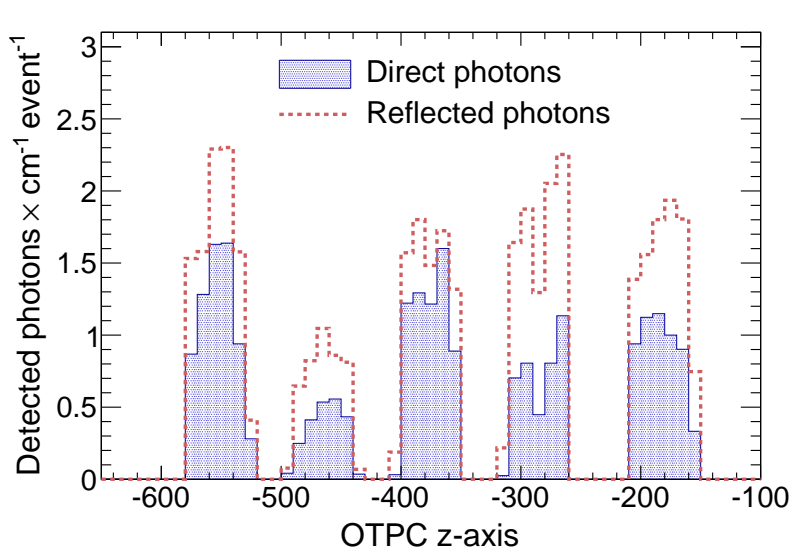

(b)

Figure 22: (a) Number of reflected Cherenkov photons per event. The direct and mirror-reflected photons are separated by applying a time-cut to the data presented in Fig. 17. The number of direct photons per event is shown in Fig. 20a. (b) Number of direct and reflected photons detected per event per $\mathrm{cm}$. The five discrete distributions along the OTPC z-axis are the 5 PM locations (left to right, PM 0 to 4).

\section{Spatial resolution}

To fully reconstruct charged particle tracks, the mirror-reflected photons are used in combination with the direct Cherenkov light. A second time cut is made on the position-corrected time, $t^{\prime}$ (Fig. 17), to isolate the reflected Cherenkov photons. A normal view time-cut of $>2.05$ ns and a stereo view time-cut of $>2.0$ ns were used on the data. A time-gap of 100 ps between the direct and reflected photon time-cuts was used to separate the two regions.

The number of reflected photons per event in the normal and stereo views is shown in Figure 22 a. The relative number of direct and reflected detected photons at each PM per event per cm is shown in Fig. 22 b. As expected from Fig. 17, there is a preponderance of reflected over direct photons.

A straight-forward way to reconstruct the spatial position of the trajectory is to take the difference of the mean direct photon arrival times and the mean reflected photon arrival times in the $t^{\prime}$ basis. Explicitly, the time difference at a PM for a given event a, $\Delta t_{P M}$, using this method is

$$
\Delta t_{P M}=\frac{1}{n} \sum_{i=1}^{n} t_{i}^{\prime \text { mirror }}-\frac{1}{m} \sum_{j=1}^{m} t_{j}^{\prime}{ }^{\text {direct }}-t_{\text {ref }}
$$

where $n$ is the number of reflected photons per event per PM and $m$ is the number of direct photons per event per PM. A time reference for the event, $t_{r e f}$ (the $R_{2}$ trigger signal time), is subtracted from each event for alignment. This time-difference measurement is inserted into Eq. 6 to obtain the lateral displacement of the particle track.

The result of Equation 9 is shown in Figure 23 when grouping together the normal view data and stereo view data separately. This result incorporates the same 522 events used in the measurement shown in Fig. 21. The normal view has an average time difference of $810 \pm 59 \mathrm{ps}$; the stereo data show a time difference of 


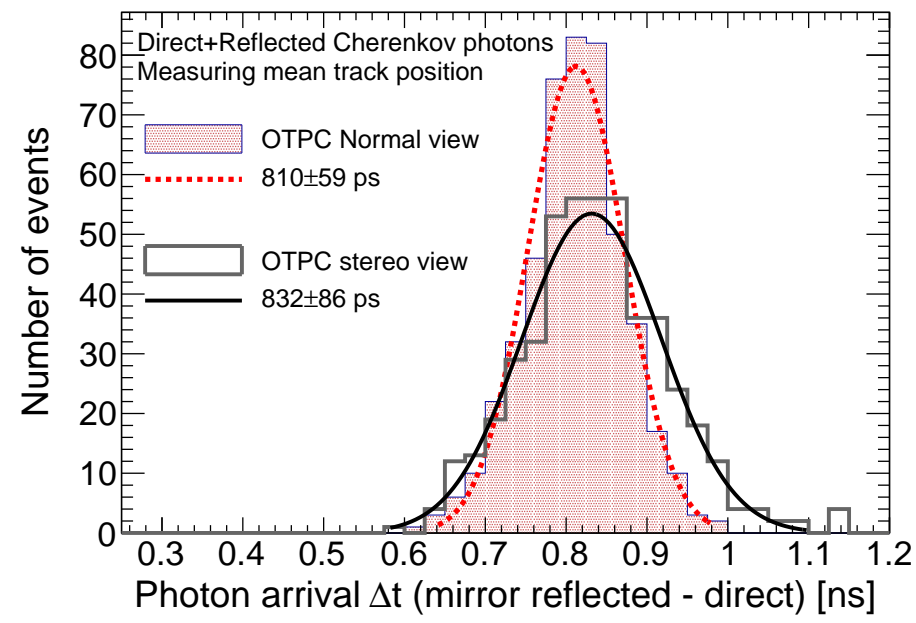

Figure 23: Time-of-arrival difference between the direct and mirror reflected Cherenkov photons for the normal and stereo views. The time differences were measured using Eq. 9. The normal view has an average time difference of $810 \pm 59$ ps and the stereo data shows a time difference of $832 \pm 86$ ps.

$832 \pm 86 \mathrm{p} \AA^{2}$. These $1 \sigma$ uncertainties correspond to spatial resolutions of $9.6 \mathrm{~mm}$ and $13.9 \mathrm{~mm}$, respectively, when combining data from the PMs.

To utilize the OTPC as a tracking detector, we use each PM to resolve the spatial coordinates along the z- and $\phi$-axes. We require that an event has more than three direct and three mirror-reflected photons for each PM. There are 127 through-going events that satisfy this condition. As expressed in Eq. 9, the radial position, $\mathrm{r}$, along the $\mathrm{z}$ and $\phi$ axes of the OTPC is defined as the displacement from the nominal beam axis. Using Eqs. 9 and 6 we can reconstruct $\mathrm{r}$ at each of the five PM positions in $\mathrm{z}$ - and $\phi$. The five distributions in the radial position are shown in Figure 24 .

The properties of the data in Figure 24 are shown in Table 1. The reconstructed radial position, r, at each $\mathrm{z}$ position is consistent with $\mathrm{r}=0$, which is the expectation with the through-going trigger. At each normal view $\mathrm{PM}$, the resolution is about $15 \mathrm{~mm}$ on this measurement. On the stereo view, the average error is $17.5 \mathrm{~mm}$. We expect the beam spatial location to have an RMS variation of $\sim 7 \mathrm{~mm}$ from the measured beam output position in the $R_{2}$ trigger.

Figure 25 shows the 3D track reconstruction of the event displayed in Figs. 14a and 18. The direct Cherenkov photons are projected onto the reconstructed radial position, $r$, and the OTPC normal and stereo-view coordinates are decomposed into the Cartesian coordinate system defined in Fig. 2

\section{Conclusions}

We have constructed and characterized the performance of a small prototype optical-TPC (OTPC), in which we measured the time and position of arrival for each individual photon emitted by Cherenkov radiation from a charged particle traversing the water volume. The photon arrival times and $2 \mathrm{D}$ spatial

\footnotetext{
${ }^{2}$ The mean time differences are slightly larger than measured on the full $16 \mathrm{GeV} / \mathrm{c}$ dataset (\$10.1 and Fig. 17 due to the event selection and the time cuts applied to separate the direct and mirror-reflected photons described in the text
} 
Table 1: Average reconstructed radial positions of tracks along OTPC z-axis. The OTPC center-line is defined as $r=0$. These data are shown in Figure 24

\begin{tabular}{cccc}
$\mathrm{PM}$ & $\bar{z}[\mathrm{~mm}]$ & $\phi$ [radians $]$ & reconstructed $\mathrm{r}[\mathrm{mm}]$ \\
\hline 0 & -550 & 0.567 & $-1.5 \pm 15.6$ \\
2 & -375 & 0.567 & $-2.75 \pm 14.8$ \\
4 & -185 & 0.567 & $-4.62 \pm 15.6$ \\
& & & \\
1 & -460 & -0.567 & $0.7 \pm 19.8$ \\
3 & -290 & -0.567 & $2.2 \pm 16.4$
\end{tabular}

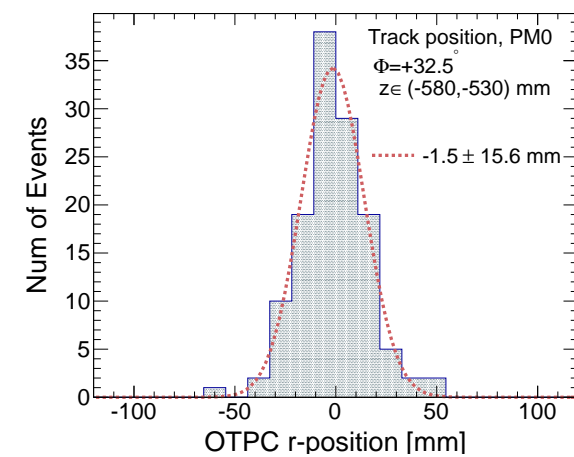

(a)

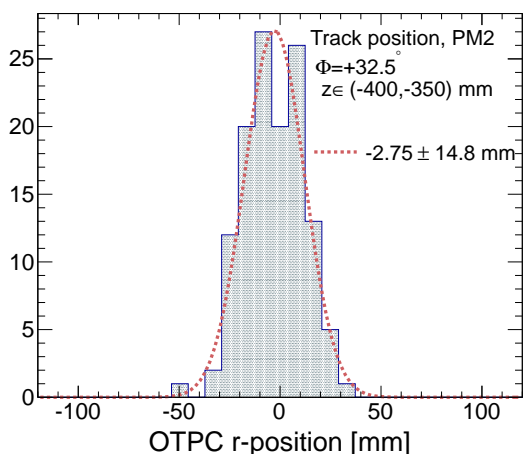

(b)

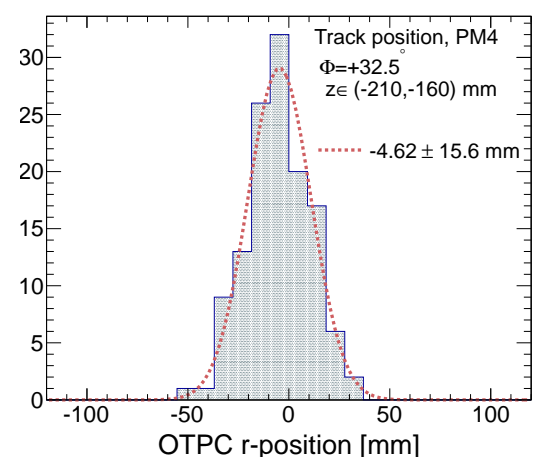

(c)

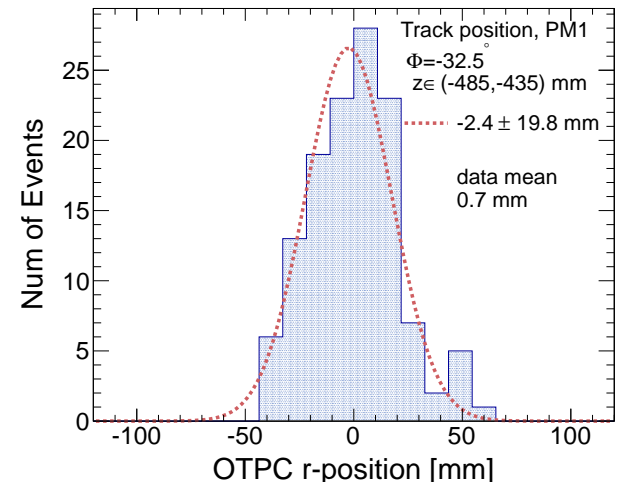

(d)

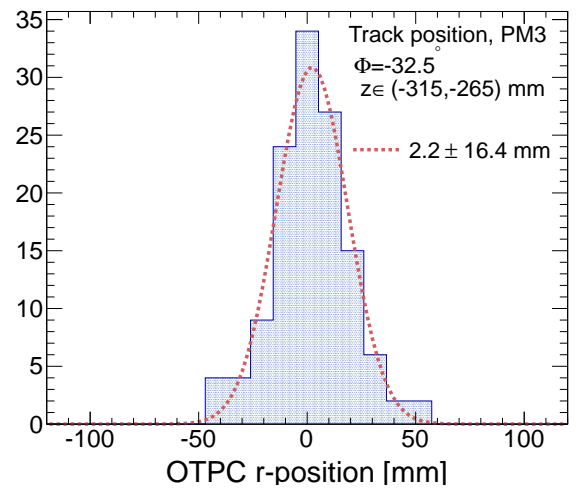

(e)

Figure 24: The reconstructed radial track position along the OTPC z-axis at each PM $\phi$-location. 


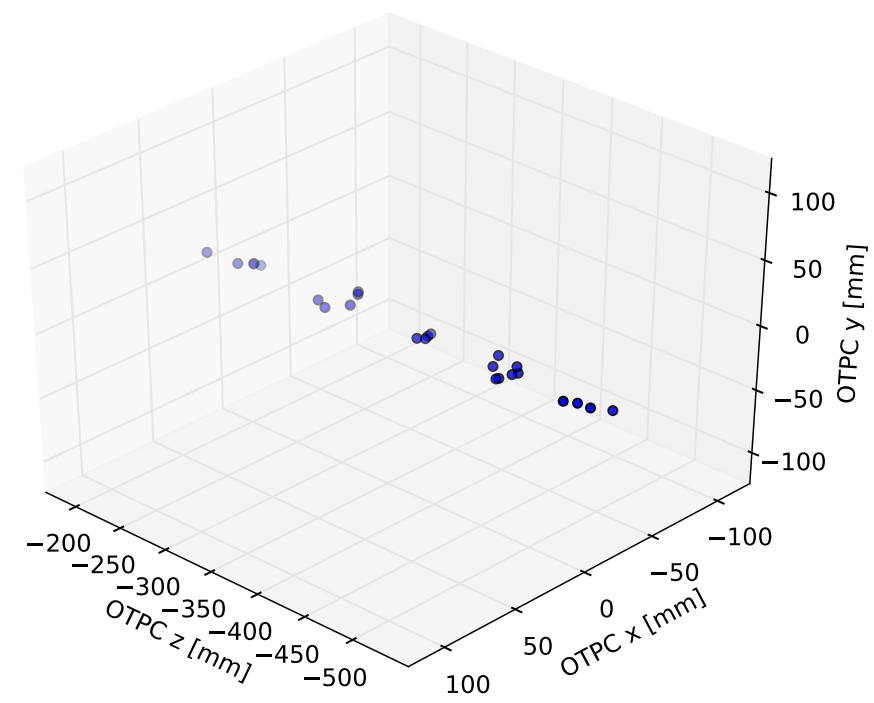

Figure 25: The 3D track reconstruction of the event shown in Figures 14a and 18. The position-corrected times, $t_{i}^{\prime}$, of the direct Cherenkov photons are projected on the reconstructed radial position (stereo, normal, $\mathrm{z})$ and decomposed into Cartesian $(\mathrm{x}, \mathrm{y}, \mathrm{z})$ coordinates.

locations are resolved to $\sim 75 \mathrm{ps}$ and $\leq 3 \times 3 \mathrm{~mm}^{2}$, respectively, using the digitized waveforms. Given the arrival positions and the drift times, the particle track position and direction can be reconstructed.

The ability of the OTPC to resolve time differences much smaller than 1 nanosecond lends itself to two strategies relevant to the economics of scale-up for larger detectors. The first was to cheaply multiply the $\sim 7 \%$ photocathode coverage on the surface of the detector by using optical mirrors; the mirrors more than doubled the OTPC light collection in our setup. For each track, the mirrors allowed for the added collection of Cherenkov photons impinging on the detector wall opposing the MCP-PMT. The reflected photons are clearly time-resolved in the data with an average delay of 770 ps compared to the direct Cherenkov light. The second strategy is to digitize the microstrips at one end of the strip with the opposing end open, so that direct pulse and the reflection from the far end provides two distinct $\sim$ ns-wide pulses per detected photon. This cuts the electronics channel count in half, and has the added advantage that the times-of-arrival at each end are measured on the same electronics channel.

For through-going tracks recorded at a secondary beam momentum of $16 \mathrm{GeV} / \mathrm{c}$, we observed $80 \pm 20$ detected Cherenkov photons per event. Each photon is resolved in time, $z$, and $\phi$, allowing a view of the 3D trajectory. We measured an angular resolution, assuming a straight track, of 60 mrad when fitting events in which more than 8 direct photons were detected among the three MCP-PMTs in the OTPC normal view. By time-resolving the direct and mirror reflected photons at $59 \mathrm{ps}$, we measure a transverse resolution on the average particle track of $\sim 10 \mathrm{~mm}$. When measuring the photon time-difference at the level of the individual PMs, we measure a track resolution of about $15 \mathrm{~mm}$ along the OTPC z-axis. We note that an entire reconstructed event takes place over a 40-cm track length and in a duration of less than 3000 
picoseconds.

\section{Acknowledgments}

We thank the staff of the Enrico Fermi Institute Electronics Development Group for their essential technical and intellectual contributions, in particular Mircea Bogdan for the design of the Central DAQ printed-circuit card, Mark Zaskowski for electronics assembly, and Mary Heintz for her superb support of the infrastructure for electronics design, computing, and web documentation. We are indebted to Richard Northrop and Robert Metz, both of the University of Chicago Physical Sciences Division Engineering Center, for expertise in mechanical design and skilled machining, respectively.

We express our gratitude to Jeffrey DeFazio, Raquel Ortega, and Emile Schyns of PHOTONIS, Inc., for providing technical details and support for the Planacon photodetectors. We thank Howard Nicholson, then of the DOE, for suggesting the use of LAPPDs for neutrino physics and for the name of the Optical Time Projection Chamber.

This work could not have been done without the support of Fermilab and, in particular, the Fermilab Test Beam operations group: Eugene Schmidt Jr., Erik Ramberg, Mandy Rominsky, and Aria Soha. Todd Nebel and Greg Sellberg provided excellent mechanical support. The LArIAT collaboration provided technical assistance and knowledge in welcoming the OTPC operation parasitically in their beam line.

This work was supported by National Science Foundation grant PHY-1066014 and Department of Energy award DE-SC0008172. Part of this work was performed at the Fermilab Test Beam Facility, which is operated by Fermi Research Alliance, LLC under Contract No. De-AC02-07CH11359 with the United Stated Department of Energy.

[1] P. A. Čerenkov. Visible radiation produced by electrons moving in a medium with velocities exceeding that of light. Phys. Rev., 52:378-379, Aug 1937.

[2] J. Beringer, et al. (PDG). Review of Particle Physics - Passage of particles through matter. Phys. Rev. D, 86:323-338, 2012.

[3] B.W. Adams, A. Elagin, H.J. Frisch, R. Obaid, E. Oberla, A. Vostrikov, R.G. Wagner, J. Wang, and M. Wetstein. Timing characteristics of large area picosecond photodetectors. Nucl.Instr.Meth A, 795:1-11, 2015.

[4] J. Milnes and J. Howorth. Picosecond time response characteristics of micro-channel plate PMT detectors. SPIE USE, $8: 89-100,2004$

[5] O.H.W. Siegmund, A.S. Tremsin, J.V. Vallerga, R. Abiad, and J. Hull. High resolution cross strip anodes for photon counting detectors. Nucl.Instr. Meth A, 504:177-181, 2003.

[6] N. Kishimoto, M. Nagamine, K. Inami, Y. Enari, and T. Ohshima. Lifetime of MCP-PMT. Nucl.Instr.Meth A, 564:204211,2006

[7] F. Tang, et al. Transmission-line readout with good time and space resolutions for Planacon MCP-PMTs. In Topical Workshop on Electronics for Particle Physics, CERN 2008, pages 579-583, 2008.

[8] H. Grabas, H.J Frisch, E. Oberla, J.-F. Genat, R. Northrop, D. McGinnis, B. Adams, M. Wetstein, R. Obaid, and F. Tang. RF Strip-Line Anodes for Psec Large-Area MCP-based Photodetectors. Nucl.Instr.Meth A, 711:123-131, 2013.

[9] B.W. Adams, A. Elagin, H.J. Frisch, R. Obaid, E. Oberla, A. Vostrikov, R.G. Wagner, and M. Wetstein. Measurements of the gain, time resolution, and spatial resolution of a $20 \times 20 \mathrm{~cm}^{2}$ MCP-based Picosecond Photo-Detector. Nucl.Instr.Meth A, 732:392, 2013 .

[10] E. Oberla, H. Grabas, J.-F. Genat, H.J. Frisch, K. Nishimura, and G. Varner. A 15 GSa/s, 1.5 GHz bandwidth waveform digitizing ASIC. Nucl.Instr.Meth A, 735:452-461, 2014

[11] J.Ahrenes et al., the AMANDA collaboration. Muon track reconstruction and data selection techniques in AMANDA. Nucl.Instr.Meth A, 524:169-194, 2004.

[12] I. Anghel, E. Catano-Mur, M.C. Sanchez, M. Wetstein, and T. Xin. Using fast photosensors in water Cherenkov neutrino detectors. DPF 2013. arXiv:1310.2654, 2013.

[13] ANNIE Collaboration. Letter of Intent: The Accelerator Neutrino Neutron Interaction Experiment (ANNIE). arXiv:1504.0180, 2015. 
[14] C. Aberle, A. Elagin, H.J. Frisch, M. Wetstein, and L. Winslow. Measuring directionality in double-beta decay and neutrino interactions with kiloton-scale scintillation detectors. Journal of Instrumentation, 9(06):P06012, 2014.

[15] LAPPD collaboration website: http://psec.uchicago.edu

[16] M.J. Minot, et al. Pilot production \& commercialization of LAPPD ${ }^{\text {TM }}$. Nucl.Instr.Meth A, 787:78-84, 2015.

[17] B.W. Adams, M. Chollet, A. Elagin, R. Obaid, E. Oberla, A. Vostrikov, M. Wetstein, and P. Webster. A test-facility for large-area microchannel plate detector assemblies using a pulsed sub-picosecond laser. Rev. Sci. Instrum., 84, 2013.

[18] PHOTONIS USA Pennsylvania, Inc. Planacon XP85022 $32 \times 32$ datasheet, Jan 2014. http://www.photonisusa.com

[19] Optical data from: (1) Enhanced Aluminum 4-6 $\lambda$ First-Surface Mirror, Edmunds Optics (2) Fused-silica glass port www.mcmaster.com (3) Optical coupling gel, code 0607, Cargille Labs. Spray paint used for light absorbing surfaces: Rustoleum, textured, indoor/outdoor, black.

[20] S. Seibert and A. LaTorre. Fast Optical Monte Carlo Simulation With Surface-Based Geometries Using Chroma. http: //chroma.bitbucket.org/, 2011.

[21] J.D. Jackson. Classical Electrodynamics. John Wiley \& Sons, Inc., 3rd edition, 1999.

[22] I. Tamm and I. Frank. Compt. Rend. Acad. Sci USSR, 14:109, 1937.

[23] M. Daimon and A. Masumura. Measurement of the refractive index of distilled water from the near-infrared region to the ultraviolet region. Appl. Opt., 46:3811-3820, 2007.

[24] Samtec, Inc. 34 AWG Micro Coax Cable Datasheet, May 2015.

[25] K. Nishimura and A. Romero-Wolf. A Correlation-Based Timing Calibration \& Diagnostic Technique for Fast Digitization ASICs. Physics Procedia, 37:1707-1714, 2012.

[26] D. Stricker-Shaver, S. Ritt, and B.J. Pichler. Novel calibration method for switched capacitor arrays enables time measurements with sub-picosecond resolution. Nuclear Science, IEEE Transactions on, 61(6):3607-3617, Dec 2014.

[27] Fermilab Test-Beam Facility. http://ppd.fnal.gov/ftbf/

[28] F. Cavanna et al. LArIAT: Liquid Argon In A Testbeam. arXiv:1406.5560, 2014.

[29] T.J. Roberts and D.M. Kaplan. G4beamline simulation program for matter-dominated beamlines. In Particle Accelerator Conference, 200\%. PAC. IEEE, pages 3468-3470, 2007.

[30] E. Oberla. Charged particle tracking in a water Cherenkov optical time-projection chamber. PhD thesis, University of Chicago, July, 2015.

[31] Advanced Laser Diode Systems. Picosecond Diode Laser - PiLas Manual and Test Report, Jun 2013. 